\title{
EXPLICIT CONTRACTION RATES FOR A CLASS OF DEGENERATE AND INFINITE-DIMENSIONAL DIFFUSIONS
}

\author{
RAPHAEL ZIMMER
}

Abstract. Given a separable and real Hilbert space $\mathbb{H}$ and a trace-class, symmetric and non-negative operator $\mathcal{G}: \mathbb{H} \rightarrow \mathbb{H}$, we examine the equation

$$
d X_{t}=-X_{t} d t+b\left(X_{t}\right) d t+\sqrt{2} d W_{t}, \quad X_{0}=x \in \mathbb{H},
$$

where $\left(W_{t}\right)$ is a $\mathcal{G}$-Wiener process on $\mathbb{H}$ and $b: \mathbb{H} \rightarrow \mathbb{H}$ is Lipschitz. We assume there is a splitting of $\mathbb{H}$ into a finite-dimensional space $\mathbb{H}^{l}$ and its orthogonal complement $\mathbb{H}^{h}$ such that $\mathcal{G}$ is strictly positive definite on $\mathbb{H}^{l}$ and the nonlinearity $b$ admits a contraction property on $\mathbb{H}^{h}$. Assuming a geometric drift condition, we derive a Kantorovich ( $L^{1}$ Wasserstein) contraction with an explicit contraction rate for the corresponding Markov kernels. Our bounds on the rate are based on the eigenvalues of $\mathcal{G}$ on the space $\mathbb{H}^{l}$, a Lipschitz bound on $b$ and a geometric drift condition. The results are derived using coupling methods.

Final version to appear in 'Stoch PDE: Anal Comp' is available at Springer via http://dx.doi.org/10.1007/s40072-017-0091-8.

\section{INTRODUCTION}

Let $(\mathbb{H},\langle\cdot, \cdot\rangle,|\cdot|)$ be a separable and real Hilbert space with inner product $\langle\cdot, \cdot\rangle$ and norm $|\cdot|$. Suppose that a trace-class, symmetric and non-negative operator $\mathcal{G}: \mathbb{H} \rightarrow \mathbb{H}$ is given. Let $\left(\mathbf{e}_{k}\right)_{k \in \mathbb{N}_{+}}$be an orthonormal basis of $\mathbb{H}$ such that for non-negative real numbers $\left(\lambda_{k}\right)$, we have $\mathcal{G} \mathbf{e}_{k}=\lambda_{k} \mathbf{e}_{k}$ and $\sum_{k=1}^{\infty} \lambda_{k}<\infty$, see e.g. 42 for the existence of such a basis. Denote by $\left(W_{t}\right)$ a $\mathcal{G}$-Wiener process on $\mathbb{H}$, i.e. $W_{t}=\sum_{k=1}^{\infty} \sqrt{\lambda_{k}} B_{t}^{k} \mathbf{e}_{k}$ for independent Brownian motions $\left(B_{t}^{k}\right)$. We consider the stochastic differential equation

$$
d X_{t}=-X_{t} d t+b\left(X_{t}\right) d t+\sqrt{2} d W_{t}, \quad X_{0}=x \in \mathbb{H},
$$

on the space $\mathbb{H}$ and assume that the non-linearity $b: \mathbb{H} \rightarrow \mathbb{H}$ is Lipschitz. In particular, there is a strong, non-explosive and continuous solution $\left(X_{t}\right)$ taking values in $\mathbb{H}$, see e.g. [34]. Moreover, $\left(X_{t}\right)$ is a Feller process and we denote the Markov transition kernels by $\left(p_{t}\right)$. Given a probability measure $\mu$ on $\mathbb{H}$, we write $\mu p_{t}(d x)=\int p_{t}(y, d x) \mu(d y)$.

Equation (11) has a natural appearance in the domain of sampling problems and acts as a diffusion limit for Markov chain Monte Carlo (MCMC) methods,

1991 Mathematics Subject Classification. 60H10, 60J25, 34F05.

Key words and phrases. stochastic differential equations, Langevin diffusion, geometric ergodicity, reflection coupling, Wasserstein distances, Kantorovich contractions.

Financial support from the German Science foundation through the Hausdorff Center for Mathematics is gratefully acknowledged. 
see [24, 38, 9, 46, 23, and the references therein. In particular, if $U: \mathbb{H} \rightarrow \mathbb{R}_{+}$ is a smooth function, if $\mathcal{G}$ is positive definite and if we choose the non-linearity $b(x)=-\mathcal{G} \nabla_{\mathbb{H}} U(x)$ in (1), then the results from [24] imply that the Markov kernels $\left(p_{t}\right)$ admit a unique invariant probability measure $\pi$ satisfying $\pi p_{t}=\pi$ for any $t \geq 0$. The measure $\pi$ is given by

$$
\pi(d x) \propto \exp (-U(x)) \mathcal{N}(0, \mathcal{G})(d x)
$$

where $\mathcal{N}(0, \mathcal{G})$ denotes a centered normal distribution on $\mathbb{H}$ with covariance operator $\mathcal{G}$. Such measures appear for example in the area of diffusion bridges, cf. [24].

Let $\mu$ be a given initial distribution. Given the outlined connection to sampling problems, an important question is whether the measure $\mu p_{t}$ converges towards $\pi$ for $t \rightarrow \infty$ in some reasonable distance and how one can obtain explicit rates for the speed of convergence. We give conditions under which the convergence takes place in Kantorovich and $L^{p}$ Wasserstein distances at an exponential rate and focus on establishing concrete bounds on the speed of convergence. Inspired by the sampling setup, we work in the following setting: Fix $n \in \mathbb{N}_{+}$. We consider a splitting of the Hilbert space $\mathbb{H}$ into a space $\mathbb{H}^{l}=\left\langle\mathbf{e}_{1}, \ldots, \mathbf{e}_{n}\right\rangle$, spanned by the first $n$ basis vectors, and its orthogonal complement $\mathbb{H}^{h}$, i.e. $\mathbb{H}=\mathbb{H}^{l} \oplus \mathbb{H}^{h}$. We call $\mathbb{H}^{l}$ low-dimensional and $\mathbb{H}^{h}$ high-dimensional space. Given $x \in \mathbb{H}$, we denote by $x^{l}$ and $x^{h}$ the orthogonal projections onto $\mathbb{H}^{l}$ and $\mathbb{H}^{h}$ respectively. Our main assumptions are:

Assumption 1. There are constants $0 \leq H_{h}<1$ and $L_{l}, L_{h}, H_{l} \geq 0$ such that

$$
\begin{aligned}
\left|b^{h}(x)-b^{h}(y)\right| & \leq H_{l}\left|x^{l}-y^{l}\right|+H_{h}\left|x^{h}-y^{h}\right| \quad \text { and } \\
\left|b^{l}(x)-b^{l}(y)\right| & \leq L_{l}\left|x^{l}-y^{l}\right|+L_{h}\left|x^{h}-y^{h}\right| \quad \text { for any } x, y \in \mathbb{H} .
\end{aligned}
$$

Assumption 2. $\mathcal{G}$ is strictly positive definite on $\mathbb{H}^{l}$, i.e. for any $k \in \mathbb{N}$ with $1 \leq k \leq n$, we have $\lambda_{k}>0$.

In the sampling setup described above, assuming that the map $x \mapsto \nabla U(x)$ is Lipschitz on $\mathbb{H}$, it is always possible to find a splitting $\mathbb{H}=\mathbb{H}^{l} \oplus \mathbb{H}^{h}$ such that Assumptions 1 and 2 are satisfied, cf. Section 3 . In addition to the above assumptions, we need some kind of localization argument, i.e. we assume either that $b$ is vanishing outside of a ball or that a geometric drift condition holds, cf. Assumptions 3 and 4 respectively. Based on these assumptions we derive quantitative Kantorovich contractions for the Markov kernels using coupling methods. The resulting contraction rates are given explicitly in terms of the eigenvalues of $\mathcal{G}$ on the space $\mathbb{H}^{l}$, the constants from Assumption 1 and the localization argument.

Outline. The main results are presented in Section 2.1. The key statements are Theorem 1 and Theorem 2. The couplings are specified in Section 2.2 and the proofs are given in Section 2.3. Applications are considered in Section 3. In the remaining part of the introduction we present additional motivation and references.

The ergodicity of degenerate and infinite-dimensional models has been extensively studied in the last two decades and by now there exists a comprehensive 
theory [19, 22, 20, 6]. Huge parts of the theory have been developed trying to answer the question, whether the $2 D$ stochastic Navier-Stokes equation is uniquely ergodic in a hypoelleptic setting, where only a few dimensions are stimulated directly with noise, cf. [18, 17]. As an intermediate step to tackle the truly hypoelleptic setting, many authors [50, 37, 36, 33, 32, 151, 2, 1] worked in an intermedium setting: They considered a splitting of the underlying Hilbert space into a finitedimensional space $\mathbb{H}^{u}$ of "unstable modes", where the dynamics is forced directly with noise, and an infinite-dimensional complement $\mathbb{H}^{s}$ of "stable modes", where the driving noise can be degenerate. Stable and unstable modes means in this context that the long time behavior of the dynamics is determined by the behavior on the space $\mathbb{H}^{u}$, cf. [16]. In this context, J.C. Mattingly proposed in [37] a coupling approach to conclude exponential mixing properties for the $2 D$ stochastic Navier-Stokes equation. In a related context, M. Hairer demonstrated in [16] the strength of asymptotic couplings to show mixing properties of degenerate systems. Finally, J.C. Mattingly and M. Hairer were able to proof the unique ergodicity of the $2 D$ Navier-Stokes equation in a hypoelleptic setting, which was a milestone in the development of ergodic theory for degenerate and infinite-dimensional systems [17, 19, 20]. Embedding some of the key concepts of the theory into a uniformly applicable framework, Mattingly, Hairer and Scheutzow developed the weak Harris theorem [22. It can be interpreted as a generalization of classical Harris type theorems [27, 39, 30, 43, 21] which have become standard tools for proving geometric ergodicity of finite-dimensional Markov processes. The weak Harris theorem further extends the range of possible applications and allows to establish geometric ergodicity under verifiable conditions. Nevertheless, being a uniform framework, applicable for a large class of Markov processes, the (weak) Harris theorem usually does not provide sharp constants for specific models and the resulting constants are often not connected to the structure of the model in a transparent way. This is due to the fact that the corresponding Kantorovich distance is usually chosen in a somehow ad hoc way, cf. [13].

In this work we do not have the aim of developing a uniform framework for various models. We focus on the very specific model (11) and establish Kantorovich contractions with explicit constants by adapting the underlying Kantorovich distance in a very specific way to the structure of the model. The approach is based on a technique from [14, 12]. Here, A. Eberle establishes Kantorovich contractions with explicit constants for finite-dimensional and non-degenerate diffusions using a combination of reflection coupling [35] and concave distance functions. While the principle idea to study Kantorovich distances w.r.t. concave underlying distances occurred at other places in the literature before [3, 22, it is noteworthy that [14, 12] presents a technique, how one can construct an explicit concave distance function which, under some reasonable assumptions, maximizes the resulting contraction rate under the reflection coupling up to constant factors. Eberle's results are based on the assumption that the underlying deterministic system of the diffusion is contractive for "large distances". In the recent work 13 this assumption is replaced by a more general Lyapunov drift condition combining Lyapunov functions with concave distance functions and reflection coupling, partially motivated 
by [22, 21]. In this work, we use the main ideas from [14, 12, 13] and extend them to the infinite-dimensional and possibly degenerate process (1) by constructing an explicit asymptotic coupling $\left(X_{t}, Y_{t}\right)$ of solutions to (1) in the sense of [16, 37], i.e. a coupling for which the processes $X_{t}$ and $Y_{t}$ converge to each other but do not necessarily meet in finite time. The Kantorovich contraction of the Markov kernels is then established by adapting the underlying cost function in a very specific way to the chosen coupling and model.

Up to the author's knowledge there are currently two works which use a reflection coupling to conclude exponential mixing properties of infinite-dimensional systems. In [5] a reflection coupling is used to prove exponential convergence for a reactiondiffusion and Burgers equation driven by space-time white noise. The article [49] makes use of an "approximated reflection coupling" to derive gradient estimates and exponential mixing for a class of non-linear monotone SPDES, where the driving noise is a $\mathcal{G}$-Wiener process, $\mathcal{G}$ being trace-class and satisfying $\langle x, \mathcal{G} x\rangle>0$ for any $x \in \mathbb{H}$. Moreover, it is assumed that the solution of the SPDE lies in the image of $\mathcal{G}$, i.e. that the equation has some kind of smoothing properties. In both articles exponential convergence in total variation norms is concluded. In contrast to these settings, we allow the operator $\mathcal{G}$ to be degenerate on the infinite-dimensional space $\mathbb{H}^{h}$ and equation (11) does not provide the additional smoothing assumed in [49]. Moreover, in our setting it is in general not true that for arbitrary $x, y \in \mathbb{H}$ we have $\left\|\delta_{x} p_{t}-\delta_{y} p_{t}\right\|_{T V} \rightarrow 0$ for $t \rightarrow \infty$, see e.g. [18, Example 3.14].

\section{MAin Results}

We present our main results. In Section 2.1 we formulate the main statements. The coupling approach leading to those statements is explained in Section 2.2. Finally, the proofs are provided in Section 2.3 .

2.1. Results. We now formulate our contraction results. As a preparation, we first introduce a norm $|\cdot|_{\alpha}$ on $\mathbb{H}$ which is equivalent to the Hilbert space norm, but has the advantage that it puts additional weight on the components in the space $\mathbb{H}^{h}$. This enables us to exploit the contraction property provided by Assumption 1. We then formulate three Kantorovich contractions with an increasing level of difficulty: In Proposition 11 we assume that the map $b$ is a contraction w.r.t. $|\cdot|_{\alpha}$ and thus a Kantorovich contraction can be established with ease. In Theorem 1 we assume that $b$ is a contraction w.r.t. $|\cdot|_{\alpha}$ only for "large distances" and adapt the underlying metric of the Kantorovich distance accordingly by involving a concave function. Finally, in Theorem [2] we replace the contraction property for large distances by a more general geometric drift condition and combine the metric considered in Theorem 1 with Lyapunov functions.

Suppose that Assumption 11 holds true. Denote

$$
\alpha=\frac{1+L_{h}}{1-H_{h}} \geq 1 \quad \text { and } \quad \beta=\alpha H_{l}+L_{l}-1 .
$$


We define a norm $|\cdot|_{\alpha}$ on $\mathbb{H}$, where the $\mathbb{H}^{h}$ component is weighted by $\alpha$ :

$$
|x|_{\alpha}=\left|x^{l}\right|+\alpha\left|x^{h}\right|
$$

Observe that $|\cdot|_{\alpha}$ is equivalent to $|\cdot|$, i.e. for any $x \in \mathbb{H}$,

$$
|x| \leq|x|_{\alpha} \leq \sqrt{2} \alpha|x|
$$

Assumption 1 implies that the non-linearity $b$ is a contraction w.r.t. $|\cdot|_{\alpha}$ in "certain regions of $\mathbb{H}^{\prime \prime}$. More precisely, we have the following statement:

Lemma 1. Assumption 1 implies the inequality

(7) $|b(x)-b(y)|_{\alpha} \leq(1+\beta)\left|x^{l}-y^{l}\right|_{\alpha}+\left(1-\frac{1}{\alpha}\right)\left|x^{h}-y^{h}\right|_{\alpha} \quad$ for any $x, y \in \mathbb{H}$.

Moreover, if $x, y \in \mathbb{H}$ satisfy

$$
(1+\beta)\left|x^{l}-y^{l}\right| \leq \frac{1}{2}\left|x^{h}-y^{h}\right|
$$

then it follows

$$
|b(x)-b(y)|_{\alpha} \leq\left(1-\frac{1}{2 \alpha}\right)|x-y|_{\alpha} .
$$

Proof. Assumption 1 implies the inequalities

$$
\begin{aligned}
|b(x)-b(y)|_{\alpha} & =\left|b^{l}(x)-b^{l}(y)\right|+\alpha\left|b^{h}(x)-b^{h}(y)\right| \\
& \leq\left(\alpha H_{l}+L_{l}\right)\left|x^{l}-y^{l}\right|+\left(H_{h}+L_{h} / \alpha\right) \alpha\left|x^{h}-y^{h}\right| \\
& =(1+\beta)\left|x^{l}-y^{l}\right|_{\alpha}+(1-1 / \alpha)\left|x^{h}-y^{h}\right|_{\alpha} .
\end{aligned}
$$

If (8) holds true, then we can further estimate:

$$
\begin{aligned}
|b(x)-b(y)|_{\alpha} & \leq\left|x^{h}-y^{h}\right|_{\alpha}-1 / 2\left|x^{h}-y^{h}\right| \\
& \leq|x-y|_{\alpha}-\left|x^{l}-y^{l}\right|-1 /(2 \alpha)\left|x^{h}-y^{h}\right|_{\alpha} \\
& \leq(1-\min \{1 /(2 \alpha), 1\})|x-y|_{\alpha} .
\end{aligned}
$$

Since $\alpha \geq 1$, we conclude that $\min \{1 /(2 \alpha), 1\}=1 /(2 \alpha)$.

Given a continuous function $d: \mathbb{H} \times \mathbb{H} \rightarrow \mathbb{R}_{+}$, the $L^{1}$ transportation cost of two Borel probability measures $\mu$ and $\nu$ on $\mathbb{H}$ w.r.t. the cost function $d$ is defined by

$$
\mathcal{W}_{d}(\nu, \mu)=\inf _{\gamma} \int d(x, y) \gamma(d x d y)
$$

where the infimum is taken over all couplings $\gamma$ with marginals $\nu$ and $\mu$ respectively. If the function $d$ is a metric, then $\mathcal{W}_{d}$ is called Kantorovich distance. Let $\mathcal{P}$ be the set of Borel probability measures on $\mathbb{H}$ with finite first moment, i.e. $\int|x| \mu(d x)<\infty$ for $\mu \in \mathcal{P}$.

If $\beta<0$, then (77) reveals that $b$ is a contraction on $\mathbb{H}$ w.r.t. $|\cdot|_{\alpha}$ which implies the following trivial result. 
Proposition 1. Let Assumption 1 be true and $\beta<0$, then

$$
\mathcal{W}_{d_{1}}\left(\mu p_{t}, \nu p_{t}\right) \leq e^{-c t} \mathcal{W}_{d_{1}}(\mu, \nu) \text { for any } \mu, \nu \in \mathcal{P} \text { and } t \geq 0 \text {, }
$$

where the distance $d_{1}$ and the rate $c$ are given by

$$
d_{1}(x, y)=|x-y|_{\alpha} \text { and } c=\min \left\{\alpha^{-1},|\beta|\right\} .
$$

The assumption $\beta<0$ implies that the underlying deterministic system of (1) is contractive and thus the statement even holds in the case $\mathcal{G} \equiv 0$. A proof using synchronous coupling is given in Section 2.3 for the readers convenience.

In order to tackle the case $\beta \geq 0$, we demand that the noise in the space $\mathbb{H}^{l}$ is non-degenerate, i.e. that Assumption 2 holds true. Moreover, we assume that $b$ is a contraction w.r.t. $|\cdot|_{\alpha}$ for "large distances". More precisely, we assume :

Assumption 3. There are $R \in(0, \infty)$ and $0 \leq M<1$ such that

$$
|b(x)-b(y)|_{\alpha} \leq M|x-y|_{\alpha} \quad \text { for any } x, y \in \mathbb{H} \text { with }|x-y|_{\alpha} \geq R .
$$

The assumption is for example satisfied, if $b$ vanishes outside of a ball. Subsequently, we will replace Assumption 3 by a more general geometric drift condition, cf. Assumption 4. Denote by $\lambda_{\star}=\min \left\{\lambda_{k}: k \in \mathbb{N}, 1 \leq k \leq n\right\}$ the smallest eigenvalue of $\mathcal{G}$ on $\mathbb{H}^{l}$. We present our first main statement.

Theorem 1. Let Assumption 1, 2, and 3 be true and assume $\beta \geq 0$. There is a distance $d_{2}$ and a constant $c \in(0, \infty)$ such that

$$
\mathcal{W}_{d_{2}}\left(\mu p_{t}, \nu p_{t}\right) \leq e^{-c t} \mathcal{W}_{d_{2}}(\mu, \nu) \quad \text { for any } \mu, \nu \in \mathcal{P} \text { and } t \geq 0 \text {. }
$$

The rate $c$ is stated explicitly in (41). In the case $\beta>0$, a lower bound is given by

$$
c \geq \frac{1}{2} \exp \left(-\frac{\beta}{8 \lambda_{\star}} R^{2}\right) \min \left\{\beta, 1-M, \frac{1}{2 \alpha}\right\} .
$$

The distance $d_{2}$ is equivalent to $|\cdot|$ and is given by

$$
d_{2}(x, y)=f\left(|x-y|_{\alpha}\right) \text {, }
$$

where $f: \mathbb{R}_{+} \rightarrow \mathbb{R}_{+}$is a strictly increasing, concave and continuous function with $f(0)=0$. The function is explicitly defined in (38). It satisfies the relations

$$
\begin{aligned}
\frac{1}{2} & \leq f^{\prime}(r) \exp \left(\frac{\beta}{8 \lambda_{\star}} r^{2}\right) \leq 1 \quad \text { for } 0<r<R \text { and } \\
f(r) & =f(R)+\frac{1}{2} \exp \left(-\frac{\beta}{8 \lambda_{\star}} R^{2}\right)(r-R) \quad \text { for } r \geq R .
\end{aligned}
$$

Theorem 1 extends ideas from [14, 12] to an infinite-dimensional and degenerate setting using asymptotic couplings in a similar spirit as [16, 37]. The proof is given in Section 2.3. The occurring factors $1 / 2$ and $1 / 8$ are, to some extend, arbitrary. Notice that the degenerate case $\left.\mathcal{G}\right|_{\mathbb{H}^{h}} \equiv 0$ is covered by the statement. Given $p \geq 1$, we write

$$
\mathcal{W}^{p}(\mu, \nu)=\left(\inf _{\gamma} \int|x-y|^{p} \gamma(d x d y)\right)^{1 / p}
$$


for the $L^{p}$ Wasserstein distance of two measures $\mu$ and $\nu$. The Kantorovich contraction (11) has several consequences. Following [12], we present some applications.

Corollary 1. There is a unique invariant probability measure $\pi \in \mathcal{P}$ such that

$$
\mathcal{W}^{1}\left(\delta_{x} p_{t}, \pi\right) \leq 4 \alpha e^{\frac{\beta R^{2}}{8 \lambda_{\star}}} e^{-c t} \mathcal{W}^{1}\left(\delta_{x}, \pi\right) \quad \text { for any } x \in \mathbb{H} \text { and } t \geq 0
$$

For measurable $g: \mathbb{H} \rightarrow \mathbb{R}$, we denote the Lipschitz constant w.r.t. $d_{2}$ by

$$
\|g\|_{\operatorname{Lip}\left(d_{2}\right)}=\sup \left\{|g(x)-g(y)| / d_{2}(x, y): x, y \in \mathbb{H}, x \neq y\right\} \text {. }
$$

Corollary 2. For any Lipschitz function $g$ and $t \geq 0$,

$$
\sup \left\{\frac{\left|\left(p_{t} g\right)(x)-\left(p_{t} g\right)(y)\right|}{|x-y|}: x, y \in \mathbb{H}, x \neq y\right\} \leq \sqrt{2} \alpha\|g\|_{\operatorname{Lip}\left(d_{2}\right)} e^{-c t} .
$$

Further consequences are discussed after Theorem 2,

We now generalize Theorem 1 and replace Assumption 3 by a geometric drift condition using arguments related to the recent work [13]. Lyapunov drift conditions are widely used to study ergodicity and stability of Markov processes, see e.g. [39, 30, 22] and the references therein. Suppose that a continuous function $V: \mathbb{H} \rightarrow[1, \infty)$ is given for which the Fréchet derivatives $\mathcal{D} V$ and $\mathcal{D}^{2} V$ exist, are continuous and bounded in bounded subsets of $\mathbb{H}$. Let

$$
\mathcal{L} V(x)=\langle\mathcal{D} V(x),-x+b(x)\rangle+\frac{1}{2} \sum_{k=1}^{\infty} \lambda_{k} \mathcal{D}^{2} V(x)\left[\mathbf{e}_{k}, \mathbf{e}_{k}\right]
$$

Assumption 4. There are constants $C, \eta \in(0, \infty)$ such that for any $x \in \mathbb{H}$,

$$
\mathcal{L} V(x) \leq C-\eta V(x) .
$$

Moreover, we assume that

$$
\lim _{r \rightarrow \infty} \inf _{|x|=r} V(x)=\infty \quad \text { and } \quad \theta=\sup _{x \in \mathbb{H}} \frac{|\mathcal{D} V(x)|}{V(x)}<\infty .
$$

The condition $\theta<\infty$ is imposed for simplicity and can be weakened. We call a function $V$ satisfying the above conditions a Lyapunov function. A typical candidate for a Lyapunov function is $V(x)=1+|x|^{2}$. Let

$$
S=\{(x, y) \in \mathbb{H} \times \mathbb{H}: V(x)+V(y)<8 C / \eta\} \quad \text { and } \quad R=\sup _{(x, y) \in S}|x-y|_{\alpha} .
$$

The set is chosen such that for any $(x, y) \notin S$,

$$
\mathcal{L} V(x)+\mathcal{L} V(y) \leq-(\eta / 2)(V(x)+V(y))-2 C .
$$

Since $V$ is bounded from below, the set $S$ cannot be empty and by continuity of $V, R>0$. Moreover, Assumption 4 implies that $R<\infty$.

Let $\mathcal{P}_{V}$ be the set of probability measures $\mu$ on $\mathbb{H}$ satisfying $\int V(x) \mu(d x)<\infty$ and write $\lambda^{\star}=\max \left\{\lambda_{k}: k \in \mathbb{N}, 1 \leq k \leq n\right\}$ for the largest eigenvalue of $\mathcal{G}$ on $\mathbb{H}^{l}$. We call a continuous function $d: \mathbb{H} \times \mathbb{H} \rightarrow[0, \infty)$ a semimetric, if it is symmetric and satisfies $d(x, y)=0$ if and only if $x=y$. We present our main result. 
Theorem 2. Let Assumptions 1, 2 and 4 be true and assume $\beta \geq 0$. There is a semimetric $d_{3}$ and a constant $c \in(0, \infty)$ such that

$$
\mathcal{W}_{d_{3}}\left(\mu p_{t}, \nu p_{t}\right) \leq e^{-c t} \mathcal{W}_{d_{3}}(\mu, \nu) \quad \text { for any } \mu, \nu \in \mathcal{P}_{V} \text { and } t \geq 0
$$

The rate $c$ is given explicitly in (61). If $\beta>0$, then a lower bound is given by

$$
c \geq \frac{1}{2} \min \left\{\exp \left(-\frac{\beta}{8 \lambda_{\star}} R^{2}-2 \theta \frac{\lambda^{\star}}{\lambda_{\star}} R\right) \min \left\{\frac{\beta}{2}, \frac{1}{4 \alpha}\right\}, \eta\right\} .
$$

The semimetric $d_{3}$ is given by

$$
d_{3}(x, y)=f\left(|x-y|_{\alpha}\right)(1+\epsilon V(x)+\epsilon V(y)),
$$

where $\epsilon \in(0, \infty)$ is a small constant. The function $f: \mathbb{R}_{+} \rightarrow \mathbb{R}_{+}$is non-decreasing, concave and continuous with $f(0)=0$. It is constant for $r \geq R$ and satisfies for $0<r<R$ the inequality

$$
\frac{1}{2} \leq f^{\prime}(r) \exp \left(\frac{\beta}{8 \lambda_{\star}} r^{2}+2 \theta \frac{\lambda^{\star}}{\lambda_{\star}} r\right) \leq 1
$$

The explicit definitions of $f$ and $\epsilon$ are given in (59) and (161) further below.

The extension of Theorem 1 to the case of a geometric drift condition is in the same spirit as in the related work [13. The multiplicative structure of $d_{3}$ is inspired by [22]. A proof of the theorem is given in Section 2.3. Notice that the function $d_{3}$ is in general not a metric, since the triangle inequality might be violated. Nevertheless, as pointed out in [22, Lemma 4.14], one can show that if the Lyapunov function $V$ growths at most exponentially in $|x|$, then $d_{3}$ satisfies a weak triangle inequality, i.e. there is $K \in(0, \infty)$ s.t. for all $x, y, z \in \mathbb{H}$ we have $d_{3}(x, y) \leq K\left[d_{3}(x, z)+d_{3}(z, y)\right]$. This is sufficient for several applications, as we discuss now. The applications are well-known in the literature.

Corollary 3. Suppose that the assumptions of Theorem 2 hold true. Let $p \geq 1$ and assume there is a constant $K \in(0, \infty)$ such that $|x-y|^{p} \leq K(V(x)+V(y))$ for any $x, y \in \mathbb{H}$. Then, the Markov kernels $\left(p_{t}\right)$ admit a unique invariant probability measure $\pi \in \mathcal{P}_{V}$ such that for any $\mu \in \mathcal{P}_{V}$ and $t \geq 0$,

$$
\mathcal{W}^{p}\left(\mu p_{t}, \pi\right)^{p} \leq 2 \exp \left(\frac{\beta}{8 \lambda_{\star}}+2 \theta \frac{\lambda^{\star}}{\lambda_{\star}}\right) \max \left\{1, \frac{K}{\epsilon \min \{1, R\}}\right\} e^{-c t} \mathcal{W}_{d_{3}}(\mu, \pi) .
$$

If $\pi$ is symmetric w.r.t. $\left(p_{t}\right)$, which is for example the case in the setting considered in Section 3.1 further below, then Corollary 3 implies a $L^{2}(\pi)$ spectral gap, cf. [23, Proposition 2.8 and Theorem 2.15] for a precise statement. A Kantorovich contraction as in Theorem 2 has further remarkable consequences: For example it allows to make statements about Markov processes which are perturbations of $\left(X_{t}\right)$, cf. e.g. [22, Section 4.1: Stability of invariant measures]. Moreover, it allows for quantifications of bias and variances of ergodic averages, cf. [28, 12, 13]. Since the latter sources do not provide statements which are directly applicable in the setting of Theorem 2, we formulate slightly adapted versions. Notice that similarly to (14) we can define $\|\cdot\|_{\operatorname{Lip}\left(d_{3}\right)}$ for the semimetric $d_{3}$. 
Corollary 4. Under the assumptions of Theorem 2, it holds

$$
\sup \left\{\frac{\left|\left(p_{t} g\right)(x)-\left(p_{t} g\right)(y)\right|}{|x-y|}: x \neq y\right\} \leq \sqrt{2} \alpha\|g\|_{\operatorname{Lip}\left(d_{2}\right)}(1+\epsilon V(x)+\epsilon V(y)) e^{-c t}
$$

for any measurable function $g$ satisfying $\|g\|_{\operatorname{Lip}\left(d_{3}\right)}<\infty$ and any $t \geq 0$.

In particular, if $x \mapsto p_{t} g(x)$ is Fréchet differentiable at some point $x \in \mathbb{H}$, then Corollary 4 provides a bound on $\left|\nabla p_{t} g(x)\right|$.

Corollary 5. Under the assumptions of Corollary 3, we have for any measurable function $g: \mathbb{H} \rightarrow \mathbb{R}$ with $\|g\|_{\operatorname{Lip}\left(d_{3}\right)}<\infty$, any $x \in \mathbb{H}$ and $t>0$,

$$
\left|E_{x}\left[\frac{1}{t} \int_{0}^{t} g\left(X_{s}\right) d s-\int g d \pi\right]\right| \leq \frac{1-e^{-c t}}{c t}\|g\|_{\operatorname{Lip}\left(d_{3}\right)} R(1+\epsilon V(x)+\epsilon C / \eta) .
$$

Corollary 6. Suppose that the assumptions of Theorem 0 hold true. Moreover, we assume that the function $x \mapsto V(x)^{2}$ satisfies the geometric drift condition

$$
\left(\mathcal{L} V^{2}\right)(x) \leq C^{\star}-\eta^{\star} V(x)^{2} \quad \text { for any } x \in \mathbb{R}^{d},
$$

with constants $C^{\star}, \eta^{\star} \in(0, \infty)$. It follows that

$$
(22) \operatorname{Cov}_{x}\left[g\left(X_{t}\right), g\left(X_{t+h}\right)\right] \mid \leq \frac{3 R^{2}}{2}\|g\|_{\operatorname{Lip}\left(d_{3}\right)}^{2}\left(1+2 \epsilon^{2}\left[C^{\star} / \eta^{\star}+e^{-\eta^{\star} t} V(x)^{2}\right]\right) e^{-c h}
$$

for any measurable function $g$ satisfying $\|g\|_{\operatorname{Lip}\left(d_{3}\right)}<\infty$ and any $t \geq 0$. In particular,

$$
\operatorname{Var}_{x}\left[\frac{1}{t} \int_{0}^{t} g\left(X_{s}\right) d s\right] \leq \frac{3 R^{2}}{c t}\|g\|_{\text {Lip }\left(d_{3}\right)}^{2}\left(1+2 \epsilon^{2}\left[C^{\star} / \eta^{\star}+e^{-\eta^{\star} t} V(x)^{2}\right]\right) .
$$

The proofs of Corollaries 4, 5] and 6] are nearly identical to the ones given in [12, 13] and are not repeated here. We remark that Theorem 2 can also be used to make statements about the existence of solutions for the poisson equation $-\mathcal{L} u=g$ associated with (1) for a certain class of functions $g$. For a precise statement regarding this topic, we refer the reader to [47, Theorem 3.1].

2.2. Couplings. We introduce the couplings used to derive upper bounds on the Kantorovich distances occurring in Proposition 1, Theorem 1 and Theorem 2.

2.2.1. Synchronous coupling. Fix initial values $\left(x_{0}, y_{0}\right) \in \mathbb{H} \times \mathbb{H}$. We call $\left(X_{t}, Y_{t}\right)$ a synchronous coupling, if it is a solution of the equation

$$
\begin{aligned}
d X_{t} & =-X_{t} d t+b\left(X_{t}\right) d t+\sqrt{2} d W_{t}, \\
d Y_{t} & =-Y_{t} d t+b\left(Y_{t}\right) d t+\sqrt{2} d W_{t}, \quad\left(X_{0}, Y_{0}\right)=\left(x_{0}, y_{0}\right)
\end{aligned}
$$

on the space $\mathbb{H} \oplus \mathbb{H}$, where $\left(W_{t}\right)$ is a $\mathcal{G}$-Wiener process on $\mathbb{H}$. The coupling is well-known and used to prove Proposition 1. 
2.2.2. Reflection coupling for non-degenerate and finite-dimensional diffusions. In order to explain the coupling leading to Theorem 1 and Theorem 2 , we shortly recall reflection coupling for non-degenerate and finite-dimensional diffusions, which goes back to [35]. We consider the following SDE in $\mathbb{R}^{d}$ :

$$
d R_{t}=a\left(R_{t}\right) d t+\sigma d B_{t}
$$

where $a: \mathbb{R}^{d} \rightarrow \mathbb{R}^{d}$ is (say) Lipschitz, $\sigma \in \mathbb{R}^{d \times d}$ satisfies $\operatorname{det}(\sigma)>0$ and $\left(B_{t}\right)$ is a $d$-dimensional Brownian motion. A reflection coupling $\left(R_{t}, S_{t}\right)$ starting at $\left(r_{0}, s_{0}\right) \in \mathbb{R}^{2 d}$ is a solution of the equation

$$
\begin{aligned}
d R_{t} & =a\left(R_{t}\right) d t+\sigma d B_{t}, \quad\left(R_{0}, S_{0}\right)=\left(r_{0}, s_{0}\right), \\
d S_{t} & =a\left(S_{t}\right) d t+\sigma\left(\mathrm{I}_{d}-2 \frac{\sigma^{-1}\left(R_{t}-S_{t}\right)}{\left|\sigma^{-1}\left(R_{t}-S_{t}\right)\right|}\left\langle\frac{\sigma^{-1}\left(R_{t}-S_{t}\right)}{\left|\sigma^{-1}\left(R_{t}-S_{t}\right)\right|}, \cdot\right)\right) d B_{t}, \quad t<T \\
S_{t} & =R_{t}, \quad t \geq T,
\end{aligned}
$$

where $T=\inf \left\{t \geq 0: X_{t}=Y_{t}\right\}$ is the coupling time. One of the crucial properties of reflection coupling is that $r_{t}=\left|R_{t}-S_{t}\right|$ satisfies almost surley the equation

$$
d\left|r_{t}\right|=r_{t}^{-1}\left\langle R_{t}-S_{t}, a\left(R_{t}\right)-a\left(S_{t}\right)\right\rangle d t+2\left|\sigma^{-1}\left(R_{t}-S_{t}\right)\right|^{-1} r_{t} d W_{t}, \quad t<T,
$$

where $\left(W_{t}\right)$ is a one-dimensional Brownian motion. We see that the driving noise $\left(W_{t}\right)$ has a direct impact on $\left|R_{t}-S_{t}\right|$, see [12] for details.

2.2.3. Switching between reflection and synchronous coupling. We present the coupling used to prove Theorem 1 and Theorem 2, Before we introduce the coupling in a rigorous way, we shortly explain the strategy: Let $\left(X_{t}, Y_{t}\right)$ be a synchronous coupling of solutions to (1), i.e. let the processes $\left(X_{t}\right)$ and $\left(Y_{t}\right)$ be driven by the same noise. We argue pathwise. Assume that $X_{t}-Y_{t}$ satisfies for some $t \geq 0$ the inequality

$$
H_{l}\left|X_{t}^{l}-Y_{t}^{l}\right| \leq\left(1-H_{h}\right)\left|X_{t}^{h}-Y_{t}^{h}\right| / 2,
$$

then Assumption 1 implies that

$$
\left|b^{h}(x)-b^{h}(y)\right| \leq H_{l}\left|X_{t}^{l}-Y_{t}^{l}\right|+H_{h}\left|X_{t}^{h}-Y_{t}^{h}\right| \leq\left(1+H_{h}\right)\left|X_{t}^{h}-Y_{t}^{h}\right| / 2,
$$

where $\left(1+H_{h}\right) / 2<1$ by assumption. In particular, as long as $X_{t}-Y_{t}$ satisfies (24), $\left|X_{t}^{h}-Y_{t}^{h}\right|$ decreases exponentially fast, while $\left|X_{t}^{l}-Y_{t}^{l}\right|$ might increase at the same time. At some point, as time increases, $X_{t}-Y_{t}$ might not satisfy (24) any more. The idea is now to use a reflection coupling of $X_{t}^{l}$ and $Y_{t}^{l}$ in the space $\mathbb{H}^{l}$ with the aim of decreasing $\left|X_{t}^{l}-Y_{t}^{l}\right|$ relative to $\left|X_{t}^{h}-Y_{t}^{h}\right|$. As soon as $X_{t}-Y_{t}$ satisfies again (24), we switch the coupling to a synchronous coupling and wait for a decrease of $\left|X_{t}^{h}-Y_{t}^{h}\right|$. If $\left|X_{t}^{h}-Y_{t}^{h}\right|$ gets again "small" compared to $\left|X_{t}^{l}-Y_{t}^{l}\right|$, we switch to a reflection coupling in $\mathbb{H}^{l}$ and so an and so forth. The coupling is visualized in Figure1. As remarked above, during the phases $X_{t}-Y_{t}$ satisfies (24), $\left|X_{t}^{l}-Y_{t}^{l}\right|$ might increase. In order to see a contraction of $X_{t}-Y_{t}$, we measure the distance with the weighted norm $|\cdot|_{\alpha}$ and replace the sector condition (24) by (8) provided by Lemma 1, Indeed, as long as $X_{t}-Y_{t}$ satisfies (8), $\left|X_{t}-Y_{t}\right|_{\alpha}$ decreases exponentially fast. This is of course not true, if $X_{t}-Y_{t}$ fails to satisfy (8)). Nevertheless, in the setting of Theorem 1, an exponential decay of $f\left(\left|X_{t}-Y_{t}\right|_{\alpha}\right)$ 


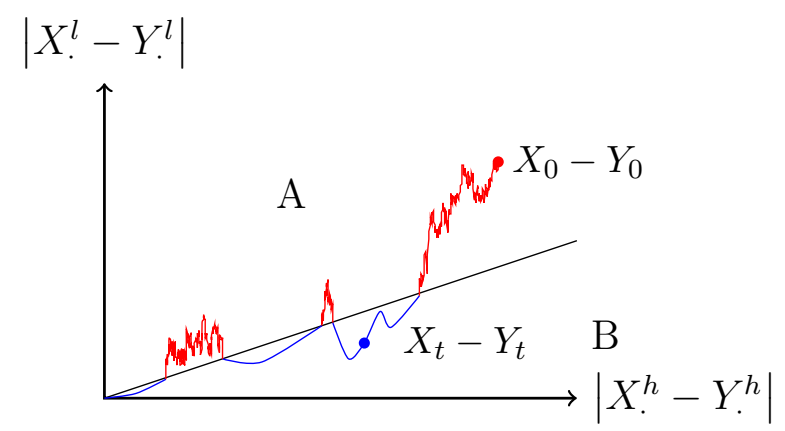

Region A

$\uparrow \quad$ reflection coupling in $\mathbb{H}^{l}$

$\longleftrightarrow$ synchronous coupling in $\mathbb{H}^{h}$

Region B

synchronous coupling in $\mathbb{H}$

FiguRE 1. Asymptotic coupling

still holds on average, if we use an appropriate concave function $f$ following [12]. The coupling strategy is similar to the ones from [16, 37]: We identify a region where the deterministic system corresponding to (1) has a contraction property and then use the available noise to drive the coupling into those regions.

We now define the coupling in a rigorous way. Fix small $\delta>0$ and denote

$$
\begin{aligned}
\mathcal{S}_{S C} & =\left\{x \in \mathbb{H}: 4(\beta+1)\left|x^{l}\right| \leq\left|x^{h}\right|\right\} \cup\left\{x \in \mathbb{H}:|x|_{\alpha} \leq \delta / 2\right\}, \\
\mathcal{S}_{R C} & =\left\{x \in \mathbb{H}: 2(\beta+1)\left|x^{l}\right| \geq\left|x^{h}\right|\right\} \cap\left\{x \in \mathbb{H}:|x|_{\alpha} \geq \delta\right\} .
\end{aligned}
$$

In comparison to the informal description further above, we add transition regions to realize transitions between the different coupling types. We describe the coupling first in words: The driving noise in the subspace $\mathbb{H}^{h}$ is always coupled synchronously, i.e. the same noise is used to drive $X_{t}^{h}$ and $Y_{t}^{h}$. In the finite-dimensional subspace $\mathbb{H}^{l}$ we use a reflection coupling of the driving noise if $X_{t}-Y_{t} \in \mathcal{S}_{R C}$ and a synchronous coupling if $X_{t}-Y_{t} \in \mathcal{S}_{S C}$. The definition of the above sets is motivated by Lemma 1. The two sets $\mathcal{S}_{R C}$ and $\mathcal{S}_{S C}$ are closed, disjoint and $\inf _{x \in \mathcal{S}_{R C}, y \in \mathcal{S}_{S C}}|x-y|>0$. The region "in between", i.e. $\mathbb{H} \backslash\left(\mathcal{S}_{R C} \cup \mathcal{S}_{S C}\right)$, is a transition region where a mixture of both couplings is used. The parameter $\delta$ occurs only for technical reasons and one should think of $\delta$ being close to 0 .

We now specify the technical realization of the coupling which follows [12, Section 6]. For given $(x, y) \in \mathbb{H} \times \mathbb{H}$, we define linear operators $R(x, y): \mathbb{H} \rightarrow \mathbb{H}$ and $S(x, y): \mathbb{H} \rightarrow \mathbb{H}$ by

$$
\begin{aligned}
& S(x, y) z=z^{h}+\operatorname{sc}(x, y) z^{l} \quad \text { and } \\
& R(x, y) z=\operatorname{rc}(x, y) z^{l} .
\end{aligned}
$$

Here sc, rc $: \mathbb{H} \oplus \mathbb{H} \rightarrow[0,1]$ are Lipschitz functions, satisfying for any $x, y \in \mathbb{H}$,

$$
\operatorname{sc}^{2}(x, y)+\operatorname{rc}^{2}(x, y)=1 \text { and } \operatorname{rc}(x, y)= \begin{cases}1 & \text { if }(x-y) \in \mathcal{S}_{R C} \\ 0 & \text { if }(x-y) \in \mathcal{S}_{S C}\end{cases}
$$

Regarding the existence of the above functions, we remark that it is enough to construct a suitable function $h: \mathbb{R}_{+} \times \mathbb{R}_{+} \rightarrow[0,1]$ such that

$$
\operatorname{rc}(x, y)=h\left(\left|x^{h}-y^{h}\right|,\left|x^{l}-y^{l}\right|\right) \quad \text { and } \quad \operatorname{sc}(x, y)=\sqrt{1-\operatorname{rc}^{2}(x, y)}
$$


satisfy the above conditions. This can be done using standard cut-off techniques. Let now $\mathbb{W}^{1}$ and $\mathbb{W}^{2}$ be independent $\mathcal{G}$-Wiener processes on $\mathbb{H}$ and fix some arbitrary unit vector $u \in \mathbb{H}^{l}$. Given starting points $\left(x_{0}, y_{0}\right) \in \mathbb{H} \times \mathbb{H}$ we define $\left(X_{t}, Y_{t}\right)_{t \geq 0}$ as a strong solution of

$d X_{t}=-X_{t} d t+b\left(X_{t}\right) d t+\sqrt{2} R\left(U_{t}\right) d \mathbb{W}_{t}^{1}+\sqrt{2} S\left(U_{t}\right) d \mathbb{W}_{t}^{2}$,

$d Y_{t}=-Y_{t} d t+b\left(Y_{t}\right) d t+\sqrt{2} \mathcal{G}^{1 / 2}\left(I-2 e_{t}\left\langle e_{t}, \cdot\right\rangle\right) \mathcal{G}^{-1 / 2} R\left(U_{t}\right) d \mathbb{W}_{t}^{1}+\sqrt{2} S\left(U_{t}\right) d \mathbb{W}_{t}^{2}$,

on $\mathbb{H} \oplus \mathbb{H}$, where $\left(X_{0}, Y_{0}\right)=\left(x_{0}, y_{0}\right), U_{t}=\left(X_{t}, Y_{t}\right)$ and

$$
e_{t}= \begin{cases}\left|\mathcal{G}^{-1 / 2}\left(X_{t}^{l}-Y_{t}^{l}\right)\right|^{-1} \mathcal{G}^{-1 / 2}\left(X_{t}^{l}-Y_{t}^{l}\right) & \text { if }\left|X_{t}^{l}-Y_{t}^{l}\right|>0 \\ u & \text { if }\left|X_{t}^{l}-Y_{t}^{l}\right|=0\end{cases}
$$

Notice that $\left|X_{t}^{l}-Y_{t}^{l}\right|=0$ implies $\operatorname{rc}\left(X_{t}, Y_{t}\right)=0$ and thus the arbitrary value $u$ in (27) is not relevant for the dynamic. The operator $\mathcal{G}^{-1 / 2}$ is well defined on the space $\mathbb{H}^{l}$ due to Assumption 2. Furthermore, by assumption, the maps $(x, y) \mapsto(b(x), b(y)),(x, y) \mapsto R(x, y)$ and $(x, y) \mapsto S(x, y)$ are Lipschitz on $\mathbb{H} \oplus \mathbb{H}$. Observe that $\left(\mathbb{W}_{t}\right)$ defined by $\mathbb{W}_{t}=\left(\mathbb{W}_{t}^{1}, \mathbb{W}_{t}^{2}\right)$ is a $\mathbb{G}$-Wiener process on $\mathbb{H} \oplus \mathbb{H}$ with $\mathbb{G}(x, y)=(\mathcal{G} x, \mathcal{G} y)$. Therefore, the above equation is a standard SDE with Lipschitz coefficients on the Hilbert space $\mathbb{H} \oplus \mathbb{H}$. The existence of a continuous, unique and non-explosive solution is well-known, see e.g. [34, Theorem 3.3]. Using the infinite-dimensional analog of Levy's characterization of Brownian motion, see e.g. [7, Theorem 4.4], and (26) one can check that

$$
\begin{aligned}
t & \mapsto \int_{0}^{t} R\left(U_{s}\right) d \mathbb{W}_{s}^{1}+\int_{0}^{t} S\left(U_{s}\right) d \mathbb{W}_{s}^{2} \text { and } \\
t & \mapsto \int_{0}^{t} \mathcal{G}^{1 / 2}\left(I-2 e_{s}\left\langle e_{s}, \cdot\right\rangle\right) \mathcal{G}^{-1 / 2} R\left(U_{s}\right) d \mathbb{W}_{s}^{1}+\int_{0}^{t} S\left(U_{s}\right) d \mathbb{W}_{s}^{2}
\end{aligned}
$$

are $\mathcal{G}$-Wiener processes on $\mathbb{H}$ and hence $\left(X_{t}, Y_{t}\right)$ is indeed a coupling.

\subsection{Proofs.}

Proof of Proposition 1. Fix initial values $x_{0}, y_{0} \in \mathbb{H}$. We first show that (10) holds for Dirac measures $\mu=\delta_{x_{0}}$ and $\nu=\delta_{y_{0}}$. Let $\left(X_{t}, Y_{t}\right)$ be a synchronous coupling as defined in Section 2.2.1. In the following, all Itô differential (in)equalities hold almost surely for all $t \geq 0$ without further mentioning.

Observe that the difference process $Z_{t}=X_{t}-Y_{t}$ satisfies the equation

$$
d Z_{t}=\left(-Z_{t}+b\left(X_{t}\right)-b\left(Y_{t}\right)\right) d s .
$$

As before, we write $Z_{t}^{l}$ and $Z_{t}^{h}$ for the orthogonal projections of $Z_{t}$ onto $\mathbb{H}^{l}$ and $\mathbb{H}^{h}$ respectively. 
Lemma 2. The processes $\left(\left|Z_{t}^{l}\right|\right)$ and $\left(\left|Z_{t}^{h}\right|\right)$ satisfy the equations

$$
\begin{aligned}
& d\left|Z_{t}^{l}\right|=I_{Z_{t}^{l} \neq 0}\left\langle\frac{Z_{t}^{l}}{\left|Z_{t}^{l}\right|},-Z_{t}+b\left(X_{t}\right)-b\left(Y_{t}\right)\right\rangle d t, \\
& d\left|Z_{t}^{h}\right|=I_{Z_{t}^{h} \neq 0}\left\langle\frac{Z_{t}^{h}}{\left|Z_{t}^{h}\right|},-Z_{t}+b\left(X_{t}\right)-b\left(Y_{t}\right)\right\rangle d t .
\end{aligned}
$$

We proof Lemma 2 further below and continue, assuming that it holds true. The coupling $\left(X_{t}, Y_{t}\right)$ yields an upper bound for the Kantorovich distance:

$$
\mathcal{W}_{d_{1}}\left(\delta_{x_{0}} p_{t}, \delta_{y_{0}} p_{t}\right) \leq E\left[\left|Z_{t}\right|_{\alpha}\right]=e^{-c t} E\left[e^{c t}\left|Z_{t}\right|_{\alpha}-\left|Z_{0}\right|_{\alpha}\right]+e^{-c t} E\left[\left|Z_{0}\right|_{\alpha}\right] \text {. }
$$

The product rule for semimartingales implies

$$
d\left(e^{c t}\left|Z_{t}\right|_{\alpha}\right)=c e^{c t}\left|Z_{t}\right|_{\alpha} d t+e^{c t} d\left|Z_{t}\right|_{\alpha} .
$$

Combining Lemma 2 and (7), we conclude that

$$
d\left|Z_{t}\right|_{\alpha} \leq\left(\beta\left|Z_{t}^{l}\right|-\alpha^{-1}\left|Z_{t}^{h}\right|_{\alpha}\right) d t \leq-c\left|Z_{t}\right|_{\alpha} d t
$$

By (31) and (32), $E\left[e^{c t}\left|Z_{t}\right|_{\alpha}-\left|Z_{0}\right|_{\alpha}\right] \leq 0$ and therefore Proposition 1 holds for Dirac measures. For the general case, let $\mu, \nu \in \mathcal{P}$. With arguments similar to [48, Theorem 4.8] one can show that for any coupling $\gamma$ of $\mu$ and $\nu$, it holds

$$
\mathcal{W}_{d_{1}}\left(\mu p_{t}, \nu p_{t}\right) \leq \int \mathcal{W}_{d_{1}}\left(\delta_{x} p_{t}, \delta_{y} p_{t}\right) \gamma(d x d y) \leq e^{-c t} \int d_{1}(x, y) \gamma(d x d y) .
$$

Taking the infimum over all couplings $\gamma$, we finish the proof of Proposition 11.

Proof of Lemma 2. We argue pathwise. The chain rule combined with (28) yields

$$
\begin{aligned}
d\left|Z_{t}^{l}\right|^{2} & =2\left\langle Z_{t}^{l},-Z_{t}+b\left(X_{t}\right)-b\left(Y_{t}\right)\right\rangle d t \\
d\left|Z_{t}^{h}\right|^{2} & =2\left\langle Z_{t}^{h},-Z_{t}+b\left(X_{t}\right)-b\left(Y_{t}\right)\right\rangle d t .
\end{aligned}
$$

We introduce a $C^{2}$ approximation of the map $t \mapsto \sqrt{t}$. Given $\epsilon>0$, we define

$$
s(r)= \begin{cases}-(1 / 8) \epsilon^{-3 / 2} r^{2}+(3 / 4) \epsilon^{-1 / 2} r+(3 / 8) \epsilon^{1 / 2} & r<\epsilon \\ \sqrt{r} & r \geq \epsilon .\end{cases}
$$

For any $r \in[0, \infty), s(r) \rightarrow \sqrt{r}$ for $\epsilon \downarrow 0$. Let $r_{t}^{l}=\left|Z_{t}^{l}\right|^{2}$. Using (33) and the chain rule, we see that for any $t \geq 0$,

$$
\begin{aligned}
s\left(r_{t}^{l}\right)-s\left(r_{0}^{l}\right) & =\int_{0}^{t} I_{r_{u}^{l} \geq \epsilon}\left\langle\frac{Z_{u}^{l}}{\left|Z_{u}^{l}\right|},-Z_{u}+b\left(X_{u}\right)-b\left(Y_{u}\right)\right\rangle d u \\
& +\int_{0}^{t} I_{0<r_{u}^{l}<\epsilon} s^{\prime}\left(r_{u}^{l}\right) 2\left\langle Z_{u}^{l},-Z_{u}+b\left(X_{u}\right)-b\left(Y_{u}\right)\right\rangle d u
\end{aligned}
$$

Observe that for $0<r_{u}^{l}<\epsilon$,

$$
\left|\left\langle X_{u}^{l}-Y_{u}^{l},-\left(X_{u}-Y_{u}\right)+b\left(X_{u}\right)-b\left(Y_{u}\right)\right\rangle\right| \leq \epsilon+\sqrt{\epsilon}\left|b\left(X_{u}\right)-b\left(Y_{u}\right)\right| .
$$

Moreover, $\sup _{u \in[0, t]}\left|b\left(X_{u}\right)-b\left(Y_{u}\right)\right|$ can be bounded by a constant, since $\left(X_{u}\right)$ and $\left(Y_{u}\right)$ are continuous and $b$ is Lipschitz. Observe that $\sup _{0 \leq r \leq \epsilon}\left|s^{\prime}(r)\right| \lesssim \epsilon^{-1 / 2}$. The 
Lebesgue dominated convergence theorem yields that the integral (37) vanishes in the limit as $\epsilon \downarrow 0$. Arguing similarly for the integral on the r.h.s. of (36), we retrieve (29). The proof of (30) is analogous.

Proof of Theorem 1. We first define the function $f$ explicitly. The function is constructed using a technique from [12, 14]. Related constructions can be found in [3, 4, 5, 13]. For real numbers $a$ and $b$, we write $a \wedge b=\min \{a, b\}$.

$$
\begin{array}{rlrl}
f(r) & =\int_{0}^{r} \phi(s \wedge R) g(s \wedge R) d s, & \Phi(r)=\int_{0}^{r} \phi(s \wedge R) d s, \\
\phi(r)=\exp \left(-\frac{\beta}{8 \lambda_{\star}} r^{2}\right), & \gamma^{-1}=\int_{0}^{R} \phi(s)^{-1} \Phi(s) d s, \\
g(r)=1-\frac{\gamma}{2} \int_{0}^{r} \phi(s)^{-1} \Phi(s) d s . & &
\end{array}
$$

We summarize important properties. The derivative of the function $f$ at $r \in(0, \infty)$ is given by the product $\phi(r \wedge R) g(r \wedge R)$. The functions $\phi$ and $g$ are strictly positive and non-increasing on $(0, R)$ and thus $f$ is strictly increasing and concave. Notice that $g(R)=1 / 2$. On the interval $[R, \infty)$ the function $f$ is linear with slope $\phi(R) / 2$. Moreover, for any $r \in(0, \infty)$,

$$
r \leq \phi(R)^{-1} \Phi(r), \quad \Phi(r) \leq r, \quad \text { and } \Phi(r) / 2 \leq f(r) \leq \Phi(r),
$$

which follows directly from the above definitions. Notice that $f(r)$ is twice continuously differentiable at $r \in(0, R)$ and that it satisfies for such $r$ the (in)equality

$$
4 \lambda_{\star} f^{\prime \prime}(r)=-\beta f^{\prime}(r) r-2 \lambda_{\star} \gamma \Phi(r) \leq-\beta f^{\prime}(r) r-2 \lambda_{\star} \gamma f(r) .
$$

We define the rate $c$ by

$$
c=\min \left\{f^{\prime}(R)(1-M), f^{\prime}(R) /(2 \alpha), 2 \lambda_{\star} \gamma\right\} .
$$

In order to see (12), observe that $f^{\prime}(R)=\phi(R) / 2$ and

$$
\gamma^{-1}=\int_{0}^{R} \phi(s)^{-1} \Phi(s) d s \leq \int_{0}^{R} \exp \left(\frac{\beta}{8 \lambda_{\star}} s^{2}\right) s d s=4 \lambda_{\star} \frac{\exp \left(\frac{\beta}{8 \lambda_{\star}} R^{2}\right)-1}{\beta} .
$$

Fix $\left(x_{0}, y_{0}\right) \in \mathbb{H} \times \mathbb{H}$. We argue that (11) holds for Dirac measures $\mu=\delta_{x_{0}}$ and $\nu=\delta_{y_{0}}$. Fix small $\delta>0$ and let $U_{t}=\left(X_{t}, Y_{t}\right)$ be the coupling with initial values $\left(x_{0}, y_{0}\right)$ defined in Section 2.2.3. We use the notation $Z_{t}=X_{t}-Y_{t}$ and $r_{t}=\left|Z_{t}\right|_{\alpha}$. The coupling yields an upper bound for the Kantorovich distance:

$$
\mathcal{W}_{d_{2}}\left(\delta_{x_{0}} p_{t}, \delta_{y_{0}} p_{t}\right) \leq E\left[f\left(r_{t}\right)\right]=e^{-c t} E\left[e^{c t} f\left(r_{t}\right)-f\left(r_{0}\right)\right]+e^{-c t} E\left[f\left(r_{0}\right)\right]
$$

We now establish bounds on $E\left[e^{c t} f\left(r_{t}\right)-f\left(r_{0}\right)\right]$. All Itô differential (in)equalities hold almost surely for all $t \geq 0$ without further mentioning. 
Lemma 3. The process $\left(r_{t}\right)$ satisfies

$$
\begin{aligned}
d r_{t} & =I_{Z_{t}^{l} \neq 0}\left\langle\frac{Z_{t}^{l}}{\left|Z_{t}^{l}\right|},-Z_{t}+b\left(X_{t}\right)-b\left(Y_{t}\right)\right\rangle d t+2 \sqrt{2} \operatorname{rc}\left(U_{t}\right) \frac{\left|Z_{t}^{l}\right|}{\left|\mathcal{G}^{-1 / 2} Z_{t}^{l}\right|} d B_{t} \\
& +\alpha I_{Z_{t}^{h} \neq 0}\left\langle\frac{Z_{t}^{h}}{\left|Z_{t}^{h}\right|},-Z_{t}+b\left(X_{t}\right)-b\left(Y_{t}\right)\right\rangle d t,
\end{aligned}
$$

where $B_{t}=\int_{0}^{t}\left\langle\mathcal{G}^{-1 / 2} e_{t}, d \mathbb{W}_{t}^{1, l}\right\rangle$ is a one-dimensional Brownian motion.

Observe that by (26) and (25), $Z_{s}^{l}=0$ implies $\operatorname{rc}\left(U_{s}\right)=0$.

Lemma 4. The process $\left(f\left(r_{t}\right)\right)$ satisfies

$$
d f\left(r_{t}\right)=f^{\prime}\left(r_{t}\right) d r_{t}+4 I_{r_{t} \neq R} f^{\prime \prime}\left(r_{t}\right) \operatorname{rc}\left(U_{t}\right)^{2}\left|Z_{t}^{l}\right|^{2}\left|\mathcal{G}^{-1 / 2} Z_{t}^{l}\right|^{-2} d t
$$

Assuming that Lemma 4 holds true, we can apply the product rule for semimartingales to conclude

$$
d\left(e^{c t} f\left(r_{t}\right)\right)=c e^{c t} f\left(r_{t}\right) d t+e^{c t} d f\left(r_{t}\right) .
$$

Lemma 5. There is a function $h: \mathbb{R}_{+} \rightarrow \mathbb{R}_{+}$with $\lim _{r \downarrow 0} h(r)=0$ such that

$$
d f\left(r_{t}\right) \leq-c f\left(r_{t}\right) d t+h(\delta) d t+f^{\prime}\left(r_{t}\right) d M_{t}^{1}
$$

where $M_{t}^{1}=\int_{0}^{t} 2 \sqrt{2} \operatorname{rc}\left(U_{t}\right)\left|Z_{t}^{l}\right|\left|\mathcal{G}^{-1 / 2} Z_{t}^{l}\right|^{-1} d B_{t}$.

Notice that $M_{t}^{1}$ is a martingale and $f^{\prime} \leq 1$. By Lemma 5, (42) and (43),

$$
\mathcal{W}_{d_{2}}\left(\delta_{x_{0}} p_{t}, \delta_{y_{0}} p_{t}\right) \leq h(\delta) / c+e^{-c t} \mathcal{W}_{d_{2}}\left(\delta_{x_{0}}, \delta_{y_{0}}\right) \text {. }
$$

Passing to the limit $\delta \rightarrow 0$, we see that (11) holds for Dirac measures. The general case can be concluded with the same argument used at the end of the proof of Proposition 1 .

Proof of Lemma 3. We first consider the projection of $Z_{t}$ onto $\mathbb{H}^{h}$. From the definition of the coupling in Section 2.2.3, we see that

$$
d Z_{t}^{h}=\left(-Z_{t}^{h}+b^{h}\left(X_{t}\right)-b^{h}\left(Y_{t}\right)\right) d t .
$$

Using the same approximation argument as in the proof Lemma 2, we conclude

$$
d\left|Z_{t}^{h}\right|=I_{Z_{t}^{h} \neq 0}\left|Z_{t}^{h}\right|^{-1}\left\langle Z_{t}^{h},-Z_{t}+b\left(X_{t}\right)-b\left(Y_{t}\right)\right\rangle d t
$$

Using the definition of the coupling in Section 2.2.3, we see that the projection of $Z_{t}$ onto $\mathbb{H}^{l}$ satisfies

$$
d Z_{t}^{l}=\left(-Z_{t}^{l}+b^{l}\left(X_{t}\right)-b^{l}\left(Y_{t}\right)\right) d t+2 \sqrt{2} \operatorname{rc}\left(U_{t}\right) \mathcal{G}^{1 / 2} e_{t}\left\langle\mathcal{G}^{-1 / 2} e_{t}, d \mathbb{W}_{t}^{1, l}\right\rangle,
$$

Notice that $B_{t}=\int_{0}^{t}\left\langle\mathcal{G}^{-1 / 2} e_{s}, d \mathbb{W}_{s}^{1, l}\right\rangle$ is a one-dimensional Brownian motion, which follows from Levy's characterization of Brownian motion. A Hilbert space version of Itô's formula, see e.g. [15, Theorem 2.9], allows to conclude

$$
\begin{aligned}
d\left|Z_{t}^{l}\right|^{2} & =2\left\langle Z_{t}^{l},-Z_{t}+b\left(X_{t}\right)-b\left(Y_{t}\right)\right\rangle d t+8 \operatorname{rc}\left(U_{t}\right)^{2}\left|\mathcal{G}^{1 / 2} e_{t}\right|^{2} d t \\
& +4 \sqrt{2} \operatorname{rc}\left(U_{t}\right)\left\langle Z_{t}^{l}, \mathcal{G}^{1 / 2} e_{t}\right\rangle d B_{t} .
\end{aligned}
$$


Given $\epsilon>0$, let $s(t)$ be the $C^{2}$ approximation of $t \mapsto \sqrt{t}$ defined in (35). Itô's formula shows

$$
\begin{aligned}
s\left(\left|Z_{t}^{l}\right|^{2}\right)-s\left(\left|Z_{0}^{l}\right|^{2}\right) & =\int_{0}^{t} s^{\prime}\left(\left|Z_{v}^{l}\right|^{2}\right) 2\left\langle Z_{v}^{l},-Z_{v}+b\left(X_{v}\right)-b\left(Y_{v}\right)\right\rangle d v \\
& +\int_{0}^{t} s^{\prime}\left(\left|Z_{v}^{l}\right|^{2}\right) 8 \operatorname{rc}\left(U_{v}\right)^{2}\left|\mathcal{G}^{1 / 2} e_{v}\right|^{2} d v \\
& +\int_{0}^{t} s^{\prime \prime}\left(\left|Z_{v}^{l}\right|^{2}\right) 16 \operatorname{rc}\left(U_{v}\right)^{2}\left(\left\langle Z_{v}^{l}, \mathcal{G}^{1 / 2} e_{v}\right\rangle\right)^{2} d v \\
& +\int_{0}^{t} s^{\prime}\left(\left|Z_{v}^{l}\right|^{2}\right) 4 \sqrt{2} \operatorname{rc}\left(U_{v}\right)\left\langle Z_{v}^{l}, \mathcal{G}^{1 / 2} e_{v}\right\rangle d B_{v}
\end{aligned}
$$

We now pass to the limit $\epsilon \downarrow 0$. The integral on the r.h.s. of (45) converges to

$$
\int_{0}^{t} I_{Z_{u}^{l} \neq 0}\left|Z_{u}^{l}\right|^{-1}\left\langle Z_{u}^{l},-Z_{u}+b\left(X_{u}\right)-b\left(Y_{u}\right)\right\rangle d u
$$

which can be argued similarly as in the proof of Lemma 2. Regarding the limits of the remaining integrals, notice that by (25) and (26),

$$
\left|Z_{t}^{l}\right|<\frac{\delta}{4} \min \left\{1, \frac{1}{4 \alpha(\beta+1)}\right\}
$$

implies $\operatorname{rc}\left(U_{t}\right)=0$. Indeed, if $\left|Z_{t}^{h}\right| \leq \delta /(4 \alpha)$ and (46) holds, then $\left|Z_{t}\right|_{\alpha}<\delta / 2$ and thus $Z_{t} \in \mathcal{S}_{\mathrm{SC}}$. If $\left|Z_{t}^{h}\right|>\delta /(4 \alpha)$ and (46) holds, then

$$
4(\beta+1)\left|Z_{t}^{l}\right|<\delta /(4 \alpha)<\left|Z_{t}^{h}\right|
$$

and thus again $Z_{t} \in \mathcal{S}_{\mathrm{SC}}$. On the other hand, $s(t)=\sqrt{t}$ for $t \geq \epsilon$, which concludes the lemma.

Proof of Lemma 4. The function $f$ can be continued to a concave function on $\mathbb{R}$ by setting $f(x)=x$ for $x<0$. The generalized Itô formula for concave functions, see e.g. [29, Thm. 22.5], implies that $\left(f\left(r_{t}\right)\right)$ satisfies the equation

$$
f\left(r_{t}\right)-f\left(r_{0}\right)=\int_{0}^{t} f_{-}^{\prime}\left(r_{s}\right) d r_{s}+\frac{1}{2} \int_{-\infty}^{\infty} L_{t}^{x} \mu_{f}(d x),
$$

where $f_{-}^{\prime}$ denotes the left-derivative of $f, \mu_{f}$ is the signed measure induced by $f_{-}^{\prime}$, i.e. $\mu_{f}[x, y)=f_{-}^{\prime}(y)-f_{-}^{\prime}(x)$ for $x \leq y$, and $L_{t}^{x}$ denotes the right-continuous local time of $\left(r_{t}\right)$. A further consequence of the generalized Itô formula is that, outside of a fixed null set, we retrieve for any measurable and non-negative function $v: \mathbb{R} \rightarrow \mathbb{R}_{+}$the equality

$$
\int_{\mathbb{R}} L_{t}^{x} v(x) d x=\int_{0}^{t} v\left(r_{s}\right) d[r]_{s} \quad \forall t \geq 0,
$$

see e.g. [29, Thm. 22.5]. Since $f^{\prime}$ exists everywhere and is continuous, we have $\mu_{f}[\{R\}]=0$. Observe that $f$ is twice continuously differentiable except at the 
point $R$. Hence by (47), (48) and Lemma 3, we can conclude that $\left(f\left(r_{t}\right)\right)$ satisfies the equations

$$
\begin{aligned}
f\left(r_{t}\right)-f\left(r_{0}\right) & =\int_{0}^{t} f^{\prime}\left(r_{s}\right) d r_{s}+\frac{1}{2} \int_{-\infty}^{\infty} I_{x \neq R} L_{t}^{x} \mu_{f}(d x), \\
\int_{-\infty}^{\infty} I_{x \neq R} L_{t}^{x} \mu_{f}(d x) & =\int_{-\infty}^{\infty} I_{x \neq R} L_{t}^{x} f^{\prime \prime}(x) d x=\int_{0}^{t} I_{r_{s} \neq R} f^{\prime \prime}\left(r_{s}\right) d[r]_{s} \\
& =\int_{0}^{t} I_{r_{s} \neq R} f^{\prime \prime}\left(r_{s}\right) 8 \operatorname{rc}\left(U_{s}\right)^{2} \frac{\left|Z_{s}^{l}\right|^{2}}{\left|\mathcal{G}^{-1 / 2} Z_{s}^{l}\right|^{2}} d s .
\end{aligned}
$$

Proof of Lemma 5. Let

$$
\begin{aligned}
w\left(U_{t}\right) & =I_{Z_{t}^{l} \neq 0}\left|Z_{t}^{l}\right|^{-1}\left\langle Z_{t}^{l},-Z_{t}+b\left(X_{t}\right)-b\left(Y_{t}\right)\right\rangle \\
& +\alpha I_{Z_{t}^{h} \neq 0}\left|Z_{t}^{h}\right|^{-1}\left\langle Z_{t}^{h},-Z_{t}+b\left(X_{t}\right)-b\left(Y_{t}\right)\right\rangle .
\end{aligned}
$$

Combining Lemma 3 and 4, we conclude

(49) $d f\left(r_{t}\right)=\left(f^{\prime}\left(r_{t}\right) w\left(U_{t}\right)+4 I_{r_{t} \neq R} f^{\prime \prime}\left(r_{t}\right) \operatorname{rc}\left(U_{t}\right)^{2} \frac{\left|Z_{t}^{l}\right|^{2}}{\left|\mathcal{G}^{-1 / 2} Z_{t}^{l}\right|^{2}}\right) d t+d M_{t}^{2}$.

with $M_{t}^{2}=\int_{0}^{t} f^{\prime}\left(r_{t}\right) d M_{t}^{1}$. Notice that for any $t \geq 0$,

$$
\left|\mathcal{G}^{-1 / 2} Z_{t}^{l}\right| \leq \lambda_{\star}^{-1 / 2}\left|Z_{t}^{l}\right|
$$

Moreover, Lemma 1 implies that

$$
w\left(U_{t}\right) \leq-r_{t}+\left|b\left(X_{t}\right)-b\left(Y_{t}\right)\right|_{\alpha} \leq-\left|Z_{t}^{h}\right|+\beta\left|Z_{t}^{l}\right| .
$$

Recall that $f$ is concave and non-decreasing. By (49), (50) and (51),

$$
\begin{aligned}
d f\left(r_{t}\right) & \leq f^{\prime}\left(r_{t}\right)\left(-r_{t}+\left|b\left(X_{t}\right)-b\left(Y_{t}\right)\right|_{\alpha}\right) d t \\
& +4 \lambda_{\star} I_{r_{t} \neq R} f^{\prime \prime}\left(r_{t}\right) \operatorname{rc}\left(U_{t}\right)^{2} d t+d M_{t}^{2} .
\end{aligned}
$$

If $r_{t} \geq R$, then Assumption 3 and (39) imply

$$
-r_{t}+\left|b\left(X_{t}\right)-b\left(Y_{t}\right)\right|_{\alpha} \leq-(1-M) r_{t} \leq-(1-M) f\left(r_{t}\right) .
$$

If $Z_{t} \notin \mathcal{S}_{\mathrm{RC}}$ and $r_{t} \geq \delta$, then by (25) and Lemma 1

$$
-r_{t}+\left|b\left(X_{t}\right)-b\left(Y_{t}\right)\right|_{\alpha} \leq-1 /(2 \alpha) r_{t} \leq-1 /(2 \alpha) f\left(r_{t}\right) .
$$

If $Z_{t} \in \mathcal{S}_{\mathrm{RC}}$ and $\delta \leq r_{t}<R$, we argue as follows: By definition we have $\operatorname{rc}\left(U_{t}\right)=1$. Lemma 1 implies the bound

$$
-r_{t}+\left|b\left(X_{t}\right)-b\left(Y_{t}\right)\right|_{\alpha} \leq \beta r_{t}
$$

Observe that for $r \in(0, R)$, inequality (40) holds true and therefore

$$
f^{\prime}\left(r_{t}\right) \beta r_{t}+4 \lambda_{\star} f^{\prime \prime}\left(r_{t}\right) \leq-2 \lambda_{\star} \gamma f\left(r_{t}\right) .
$$

Recall (38) and (39) to see that if $r_{t} \leq \delta$ holds, then we can estimate

$$
f^{\prime}\left(r_{t}\right) \beta r_{t} \leq \beta \delta \quad \text { and } \quad f\left(r_{t}\right) \leq r_{t} \leq \delta .
$$


Combining (52), (53), (54), (55), (56), (57) and (41), we conclude

$$
d f\left(r_{t}\right) \leq-c f\left(r_{t}\right) d t+(c+\beta) \delta d t+d M_{t}^{2} .
$$

The claim follows by setting $h(\delta)=(c+\beta) \delta$.

Proof of Corollary 1. By (39), (11) and (6) , we conclude for any $x, y \in \mathbb{H}$,

$$
\mathcal{W}^{1}\left(\delta_{x} p_{t}, \delta_{y} p_{t}\right) \leq 2 \phi(R)^{-1} \mathcal{W}_{d_{2}}\left(\delta_{x} p_{t}, \delta_{y} p_{t}\right) \leq 4 \alpha \phi(R)^{-1} e^{-c t} \mathcal{W}^{1}\left(\delta_{x}, \delta_{y}\right) .
$$

The fact that the Markov kernels $\left(p_{t}\right)$ admit a unique invariant measure $\pi$ satisfying $\pi p_{t}=\pi$ for any $t \geq 0$ now follows by standard arguments, see e.g. [12, Corollary $2.5]$.

Proof of Corollary 2. The proof follows [12, Section 4]. Let $\left(X_{t}\right)$ be a solution of (1) with $X_{0}=x$. Assumption 3 implies that the first moments of $X_{t}$ are uniformly bounded in time, i.e. $\sup _{t>0} E_{x}\left[\left|X_{t}\right|\right]<\infty$. In particular, for any $x \in \mathbb{H}, t \geq 0$ and any Lipschitz function $g, \int g(y) p_{t}(x, d y)<\infty$. Fix $x, y \in \mathbb{H}$ and let $\left(X_{t}, Y_{t}\right)$ be any coupling of $\delta_{x} p_{t}$ and $\delta_{y} p_{t}$. It follows

$$
\left|\left(p_{t} g\right)(x)-\left(p_{t} g\right)(y)\right| \leq E\left[\left|g\left(X_{t}\right)-g\left(Y_{t}\right)\right|\right] \leq\|g\|_{\operatorname{Lip}\left(d_{2}\right)} E\left[d_{2}\left(X_{t}, Y_{t}\right)\right],
$$

and hence by (11), (6) and (39),

$$
\left|\left(p_{t} g\right)(x)-\left(p_{t} g\right)(y)\right| \leq\left\|g||_{\operatorname{Lip}\left(d_{2}\right)} e^{-c t} f\left(|x-y|_{\alpha}\right) \leq \sqrt{2} \alpha\right\| g \|_{\operatorname{Lip}\left(d_{2}\right)} e^{-c t}|x-y| .
$$

Proof of Theorem 2. The proof is close to the proof of Theorem 1. We use again the coupling from Section 2.2.3, but use a slightly different function $f$.

$$
\begin{array}{ll}
f(r)=\int_{0}^{r \wedge R} \phi(s) g(s) d s & \Phi(r)=\int_{0}^{r \wedge R} \phi(s) d s \\
\phi(r)=\exp \left(-\frac{\beta}{8 \lambda_{\star}} r^{2}-2 \theta \frac{\lambda^{\star}}{\lambda_{\star}} r\right) & \gamma^{-1}=\int_{0}^{R} \Phi(s) \phi(s)^{-1} d s \\
g(r)=1-\frac{\gamma}{2} \int_{0}^{r \wedge R} \Phi(s) \phi(s)^{-1} d s &
\end{array}
$$

We highlight the differences to the situation in Theorem 1. This time, $f$ is constant on $[R, \infty)$ and it is not differentiable at the point $R$. Nevertheless, it is concave and the left-derivative $f_{-}^{\prime}$ exists everywhere. Observe that the inequalities (39) still hold true on the interval $[0, R]$. Moreover, the function $f$ is twice continuously differentiable on $(0, R)$ and satisfies there the (in)equality

$$
\begin{aligned}
4 \lambda_{\star} f^{\prime \prime}(r) & =-f^{\prime}(r)\left(\beta r+8 \theta \lambda^{\star}\right)-2 \lambda_{\star} \gamma \Phi(r) \\
& \leq-f^{\prime}(r)\left(\beta r+8 \theta \lambda^{\star}\right)-2 \lambda_{\star} \gamma f(r) .
\end{aligned}
$$

The contraction rate $c$ in (19) and the constant $\epsilon$ in (21) are given by

$$
c=\min \left\{\lambda_{\star} \gamma, \frac{\phi(R)}{8 \alpha}, \frac{\eta}{2}\right\} \quad \text { and } 2 C \epsilon=\min \left\{\lambda_{\star} \gamma, \frac{\phi(R)}{8 \alpha}\right\} \geq c .
$$

The lower bound (201) can be derived similarly as in the proof of Theorem 1, 
Fix small $\delta>0$, initial conditions $\left(x_{0}, y_{0}\right) \in \mathbb{H} \times \mathbb{H}$ and let $U_{t}=\left(X_{t}, Y_{t}\right)$ be the coupling defined in Section 2.2.3. We use the notation

$$
\begin{aligned}
Z_{t} & =X_{t}-Y_{t}, & r_{t} & =\left|Z_{t}\right|_{\alpha}, \\
G(x, y) & =1+\epsilon V(x)+\epsilon V(y) & Q_{t} & =f\left(r_{t}\right) G\left(X_{t}, Y_{t}\right) .
\end{aligned}
$$

The coupling yields an upper bound for the Kantorovich distance:

$$
\mathcal{W}_{d_{3}}\left(\delta_{x_{0}} p_{t}, \delta_{y_{0}} p_{t}\right) \leq E\left[Q_{t}\right]=e^{-c t} E\left[e^{c t} Q_{t}-Q_{0}\right]+e^{-c t} E\left[Q_{0}\right]
$$

We now estimate $E\left[e^{c t} Q_{t}-Q_{0}\right]$ and proceed similarly to the proof of Theorem 1 , Observe that Lemma 3 still holds true, since we use the same coupling as in the proof of Theorem 1 .

Lemma 6. The process $\left(f\left(r_{t}\right)\right)$ satisfies

$$
\begin{aligned}
d f\left(r_{t}\right) & =f_{-}^{\prime}\left(r_{t}\right) d r_{t}+\frac{1}{2} \int_{-\infty}^{\infty} L_{t}^{x} \mu_{f}(d x) \\
& \leq f_{-}^{\prime}\left(r_{t}\right) d r_{t}+4 I_{r_{t} \neq R} f^{\prime \prime}\left(r_{t}\right) \operatorname{rc}\left(U_{t}\right)^{2}\left|Z_{t}^{l}\right|^{2}\left|\mathcal{G}^{-1 / 2} Z_{t}^{l}\right|^{-2} d t
\end{aligned}
$$

The notation $\mu_{f}$ and $L_{t}^{x}$ is defined in the proof of Lemma 4 .

Lemma 7. The process $\left(G\left(X_{t}, Y_{t}\right)\right)$ satisfies

$$
d G\left(X_{t}, Y_{t}\right)=\epsilon\left(\mathcal{L} V\left(X_{t}\right)+\mathcal{L} V\left(Y_{t}\right)\right) d t+d M_{t}^{3},
$$

where $\left(M_{t}^{3}\right)$ is a local martingale given by

$$
\begin{aligned}
d M_{t}^{3} & =\sqrt{2} \epsilon\left\langle\mathcal{D} V\left(X_{t}\right)+\mathcal{D} V\left(Y_{t}\right), d \mathbb{W}_{t}^{2, h}\right\rangle \\
& +\sqrt{2} \epsilon \operatorname{sc}\left(U_{t}\right)\left\langle\mathcal{D} V\left(X_{t}\right)+\mathcal{D} V\left(Y_{t}\right), d \mathbb{W}_{t}^{2, l}\right\rangle \\
& +\sqrt{2} \epsilon \operatorname{rc}\left(U_{t}\right)\left\langle\mathcal{D} V\left(X_{t}\right)+\mathcal{D} V\left(Y_{t}\right), d \mathbb{W}_{t}^{1, l}\right\rangle \\
& -2 \sqrt{2} \epsilon \operatorname{rc}\left(U_{t}\right)\left\langle\mathcal{D} V\left(Y_{t}\right), \mathcal{G}^{1 / 2} e_{t}\right\rangle\left\langle\mathcal{G}^{-1 / 2} e_{t}, d \mathbb{W}_{t}^{1, l}\right\rangle
\end{aligned}
$$

The product rule for semimartingales implies

$$
d\left(e^{c t} Q_{t}\right)=c e^{c t} Q_{t} d t+e^{c t} d Q_{t} .
$$

Lemma 8. There is $h: \mathbb{R}_{+} \rightarrow \mathbb{R}_{+}$with $\lim _{r \downarrow 0} h(r)=0$ such that

$$
d Q_{t} \leq-c Q_{t} d t+\left(1+\epsilon V\left(X_{t}\right)+\epsilon V\left(Y_{t}\right)\right) h(\delta) d t+d M_{t}^{4},
$$

where $M_{t}^{4}=\int_{0}^{t} f\left(r_{s}\right) d M_{s}^{3}+\int_{0}^{t} G\left(X_{s}, Y_{s}\right) f_{-}^{\prime}\left(r_{s}\right) d M_{s}^{1}$ is a local martingale.

The martingale $\left(M_{t}^{1}\right)$ is defined in Lemma 5 .

Lemma 9. For any $t \geq 0$, there is $K_{t} \in(0, \infty)$, not depending on $\delta$, such that

$$
E\left[e^{c t} Q_{t}-Q_{0}\right] \leq K_{t} h(\delta)
$$


Combining Lemma 9 with (62) yields

$$
\mathcal{W}_{d_{3}}\left(\delta_{x_{0}} p_{t}, \delta_{y_{0}} p_{t}\right) \leq K_{t} h(\delta)+e^{-c t} \mathcal{W}_{d_{3}}\left(\delta_{x_{0}}, \delta_{y_{0}}\right)
$$

Passing to the limit $\delta \rightarrow 0$, we see that (19) holds for Dirac measures. The general case can be concluded with the same argument used at the end of the proof of Proposition 1.

Proof of Lemma 6. The proof is analogous to the proof of Lemma 4, except that now $f$ is not continuously differentiable everywhere. In particular, $f_{-}^{\prime}$ has a discontinuity at the point $R$ and therefore we do not have $\mu_{f}[\{R\}]=0$. Nevertheless, since $f$ is concave we know that $\mu_{f}[\{R\}]<0$.

Proof of Lemma 7. The assumptions imposed on $V$ allow to apply Itô's formula in a Hilbert space setting, see e.g. [15, Theorem 2.9]. Recalling the definition of the coupling from Section 2.2.3, we see that (63) holds true.

Proof of Lemma 8. The product rule for semimartingales implies that $\left(Q_{t}\right)$ satisfies (66) $d Q_{t}=G\left(X_{t}, Y_{t}\right) d f\left(r_{t}\right)+f\left(r_{t}\right) d G\left(X_{t}, Y_{t}\right)+d\left[f\left(r_{.}\right), G\left(X ., Y_{.}\right)\right]_{t}$,

where $[\cdot, \cdot]$ denotes the quadratic variation. By Lemma 6, (50) and (51),

$$
\begin{aligned}
G\left(X_{t}, Y_{t}\right) d f\left(r_{t}\right) & \leq G\left(X_{t}, Y_{t}\right) f_{-}^{\prime}\left(r_{t}\right)\left(-\left|Z_{t}^{h}\right|+\beta\left|Z_{t}^{l}\right|\right) d t \\
& +G\left(X_{t}, Y_{t}\right) 4 \lambda_{\star} I_{r_{t} \neq R} f^{\prime \prime}\left(r_{t}\right) \operatorname{rc}\left(U_{t}\right)^{2} d t+d M_{t}^{5},
\end{aligned}
$$

where $M_{t}^{5}=\int_{0}^{t} G\left(X_{s}, Y_{s}\right) f_{-}^{\prime}\left(r_{s}\right) d M_{s}^{1}$ is a local martingale.

Lemma 7 and Assumption 4 imply that

$$
f\left(r_{t}\right) d G\left(X_{t}, Y_{t}\right) \leq f\left(r_{t}\right) \epsilon\left(2 C-\eta\left(V\left(X_{t}\right)+V\left(Y_{t}\right)\right)\right) d t+d M_{t}^{6},
$$

with $M_{t}^{6}=\int_{0}^{t} f\left(r_{t}\right) d M_{t}^{3}$. Using Lemma 3, 6 and 17, we establish the bound

$$
\begin{aligned}
{[f(r .), G(X ., Y .)]_{t} } & =4 \epsilon \int_{0}^{t} f_{-}^{\prime}\left(r_{s}\right) \operatorname{rc}\left(U_{s}\right)^{2} \frac{\left|Z_{s}^{l}\right|}{\left|\mathcal{G}^{-1 / 2} Z_{s}^{l}\right|^{2}}\left\langle\mathcal{D} V\left(X_{s}\right)-\mathcal{D} V\left(Y_{s}\right), Z_{s}^{l}\right\rangle d s \\
& \leq 4 \epsilon \lambda^{\star} \int_{0}^{t} f_{-}^{\prime}\left(r_{s}\right) \operatorname{rc}\left(U_{s}\right)^{2}\left(\left|\mathcal{D} V\left(X_{s}\right)\right|+\left|\mathcal{D} V\left(Y_{s}\right)\right|\right) d s
\end{aligned}
$$

where $\lambda^{\star}$ is the largest eigenvalue of $\mathcal{G}$ on $\mathbb{H}^{l}$. Assumption 4 implies

$$
[f(r .), G(X ., Y .)]_{t} \leq 8 \theta \lambda^{\star} \int_{0}^{t} f_{-}^{\prime}\left(r_{s}\right) \operatorname{rc}\left(U_{s}\right)^{2} G\left(X_{s}, Y_{s}\right) d s
$$

Combining (66), (67), (68) and (69), we conclude that

$$
\begin{aligned}
d Q_{t} & \leq G\left(X_{t}, Y_{t}\right) f_{-}^{\prime}\left(r_{t}\right)\left(-\left|Z_{t}^{h}\right|+\beta\left|Z_{t}^{l}\right|+8 \theta \lambda^{\star} \operatorname{rc}\left(U_{t}\right)^{2}\right) d t \\
& +G\left(X_{t}, Y_{t}\right) 4 \lambda_{\star} I_{r_{t} \neq R} f^{\prime \prime}\left(r_{t}\right) \operatorname{rc}\left(U_{t}\right)^{2} d t \\
& +f\left(r_{t}\right) \epsilon\left(2 C-\eta\left(V\left(X_{t}\right)+V\left(Y_{t}\right)\right)\right) d t+d M_{t}^{5}+d M_{t}^{6} .
\end{aligned}
$$

We are now in a position to argue (65) and do a pathwise case distinction:

If $r_{t}>R$, then $\left(X_{t}, Y_{t}\right) \notin S$ by (17). By (18) and (61),

$$
\begin{aligned}
f\left(r_{t}\right) \epsilon\left(2 C-\eta\left(V\left(X_{t}\right)+V\left(Y_{t}\right)\right)\right) & \leq f\left(r_{t}\right)\left(-2 C \epsilon-\eta / 2\left(\epsilon V\left(X_{t}\right)+\epsilon V\left(Y_{t}\right)\right)\right) \\
& \leq-c f\left(r_{t}\right) G\left(X_{t}, Y_{t}\right)=-c Q_{t} .
\end{aligned}
$$


Moreover, $f$ is constant on $(R, \infty)$ and thus $f^{\prime}\left(r_{t}\right)=f^{\prime \prime}\left(r_{t}\right)=0$.

Now assume that $\delta \leq r_{t} \leq R$ and $Z_{t} \in \mathcal{S}_{\mathrm{RC}}$. By (26), we have that $\operatorname{rc}\left(U_{t}\right)=1$. Notice that equality (48) implies for any fixed $t \geq 0$,

$$
\lambda_{\text {Leb }}\left(\left\{0 \leq s \leq t: r_{s}=R \text { and } \operatorname{rc}\left(U_{s}\right)>0\right\}\right)=0,
$$

i.e. the Lebesgue measure of the time $\left(r_{s}\right)$ spends at the point $R$ up to time $t$, while $\operatorname{rc}\left(U_{s}\right)>0$ is almost surely zero. This allows us to neglect the case $r_{t}=R$. Moreover, $f$ is twice continuously differentiable on $(0, R)$ and fulfils inequality (60). We conclude that for $\delta \leq r_{t}<R$ with $Z_{t} \in \mathcal{S}_{\mathrm{RC}}$,

$$
\begin{aligned}
& G\left(X_{t}, Y_{t}\right) f^{\prime}\left(r_{t}\right)\left(\beta\left|Z_{t}^{l}\right|+8 \theta \lambda^{\star}\right)+G\left(X_{t}, Y_{t}\right) 4 \lambda_{\star} f^{\prime \prime}\left(r_{t}\right)+f\left(r_{t}\right) \epsilon 2 C \\
\leq & -2 \lambda_{\star} \gamma G\left(X_{t}, Y_{t}\right) f\left(r_{t}\right)+f\left(r_{t}\right) \epsilon 2 C \leq-c Q_{t},
\end{aligned}
$$

where we used (61) and $G \geq 1$.

If $\delta \leq r_{t} \leq R$ and $Z_{t} \notin \mathcal{S}_{\mathrm{RC}}$, then by (25), $2(\beta+1)\left|Z_{t}^{l}\right| \leq\left|Z_{t}^{h}\right|$, but we do not necessarily have $\operatorname{rc}\left(U_{t}\right)=1$. Nevertheless, (70) is still true and (60) implies

$$
f_{-}^{\prime}\left(r_{t}\right) 8 \theta \lambda^{\star} \operatorname{rc}\left(U_{t}\right)^{2}+4 \lambda_{\star} f^{\prime \prime}\left(r_{t}\right) \operatorname{rc}\left(U_{t}\right)^{2} \leq 0 \quad \text { for } 0<r_{t}<R .
$$

Lemma 1 shows that

$$
-\left|Z_{t}^{h}\right|+\beta\left|Z_{t}^{l}\right| \leq-1 /(2 \alpha) r_{t} \leq-1 /(2 \alpha) f\left(r_{t}\right)
$$

and thus

$$
\begin{aligned}
& G\left(X_{t}, Y_{t}\right) f_{-}^{\prime}\left(r_{t}\right)\left(-\left|Z_{t}^{h}\right|+\beta\left|Z_{t}^{l}\right|\right)+f\left(r_{t}\right) \epsilon 2 C \\
\leq \quad & -\phi(R) /(4 \alpha) Q_{t}+f\left(r_{t}\right) \epsilon 2 C \leq-c Q_{t},
\end{aligned}
$$

where we used (61) and the fact that $f^{\prime}$ is non-negative and decreasing on $(0, R)$ with $f_{-}^{\prime}(R)=\phi(R) / 2$.

Now assume $r_{t} \leq \delta$. Similarly to the last case, (171) holds true. Since $f_{-}^{\prime} \leq 1$ and $f(r) \leq r$, we can estimate

$$
G\left(X_{t}, Y_{t}\right) f_{-}^{\prime}\left(r_{t}\right) \beta\left|Z_{t}^{l}\right|+f\left(r_{t}\right) \epsilon 2 C \leq G\left(X_{t}, Y_{t}\right)(\beta+2 C \epsilon) \delta
$$

We conclude the lemma setting $h(\delta)=(c+\beta+2 C \epsilon) \delta$.

Proof of Lemma 9. We introduce stopping times

$$
\begin{aligned}
T & =\inf \left\{t \geq 0: X_{t}=Y_{t}\right\} \quad \text { and } \\
T_{m} & =\inf \left\{t \geq 0:\left|X_{t}-Y_{t}\right| \leq 1 / m \text { or } \max \left\{\left|X_{t}\right|,\left|Y_{t}\right|\right\} \geq m\right\} .
\end{aligned}
$$

Since the process $\left(X_{t}, Y_{t}\right)$ is non-explosive, we have $T_{m} \uparrow T$ for $m \uparrow \infty$. We get

$$
\begin{aligned}
E\left[e^{c t} Q_{t}\right] & =E\left[e^{c t} Q_{t} I_{t<T}\right]=\lim _{m \rightarrow \infty} E\left[e^{c t \wedge T_{m}} Q_{t \wedge T_{m}} I_{t<T_{m}}\right] \\
& \leq \liminf _{m \rightarrow \infty} E\left[e^{c t \wedge T_{m}} Q_{t \wedge T_{m}}\right] .
\end{aligned}
$$


Fix $m \in \mathbb{N}$ and notice that the stopped process $\left(M_{t \wedge T_{m}}^{4}\right)$ defined in Lemma 8 is a martingale. Using (64) and Lemma 8, we conclude

$$
\begin{aligned}
E\left[e^{c\left(t \wedge T_{m}\right)} Q_{t \wedge T_{m}}-Q_{0}\right] & \leq E\left[\int_{0}^{t \wedge T_{m}} e^{c s} G\left(X_{s}, Y_{s}\right) d s\right] h(\delta) \\
& \leq E\left[\int_{0}^{t} e^{c s} G\left(X_{s}, Y_{s}\right) d s\right] h(\delta) .
\end{aligned}
$$

Assumption 4 implies that there is a constant $A \in(0, \infty)$ such that

$$
\sup _{t \in[0, \infty)}\left(E\left[V\left(X_{t}\right)\right]+E\left[V\left(Y_{t}\right)\right]\right)<A .
$$

Proof of Corollary [3. Let $x, y \in \mathbb{H}$ with $|x-y|_{\alpha} \leq \min \{1, R\}$. By (66) and (39),

$$
|x-y|^{p} \leq|x-y|_{\alpha} \leq 2 \phi^{-1}(\min \{1, R\}) f\left(|x-y|_{\alpha}\right)(1+\epsilon V(x)+\epsilon V(y)) .
$$

On the other hand, if $|x-y|_{\alpha}>\min \{1, R\}$, then we get

$$
|x-y|^{p} \leq K(V(x)+V(y)) \leq \frac{K}{\epsilon f(\min \{1, R\})} f\left(|x-y|_{\alpha}\right)(1+\epsilon V(x)+\epsilon V(y)) .
$$

By (39),$f(\min \{1, R\}) \geq \Phi(\min \{1, R\}) / 2 \geq \min \{1, R\} \phi(\min \{1, R\}) / 2$. Combining the bounds, we get for any $x, y \in \mathbb{H}$,

$$
|x-y|^{p} \leq 2 \phi^{-1}(\min \{1, R\}) \max \left\{1, \frac{K}{\epsilon \min \{1, R\}}\right\} d_{3}(x, y) .
$$

Using (73) and Theorem 2, we can conclude that

$$
(74) \mathcal{W}^{p}\left(\mu p_{t}, \nu p_{t}\right)^{p} \leq 2 \phi^{-1}(\min \{1, R\}) \max \left\{1, \frac{K}{\epsilon \min \{1, R\}}\right\} e^{-c t} \mathcal{W}_{d_{3}}(\mu, \nu)
$$

for any $\mu, \nu \in \mathcal{P}_{V}$ and $t \geq 0$. Notice that Assumption 4 implies that

$$
\sup _{t \geq 0} \int V(y)\left(\delta_{x} p_{t}\right)(d y)<\infty \quad \text { for any } x \in \mathbb{H} \text {. }
$$

In particular, (74) and (75) together imply that there is a constant $C \in(0, \infty)$ such that

$$
\mathcal{W}^{p}\left(\delta_{x} p_{m}, \delta_{x} p_{n}\right)^{p}=\mathcal{W}^{p}\left(\delta_{x} p_{m-n} p_{n}, \delta_{x} p_{n}\right)^{p} \leq C e^{-c n}
$$

for any integers $m>n>0$. We see that $\left(\delta_{x} p_{n}\right)_{n \in \mathbb{N}}$ is a Cauchy sequence w.r.t. $\mathcal{W}^{p}$. Moreover, the $L^{p}$ Wasserstein space is Polish and convergence w.r.t. $\mathcal{W}^{p}$ implies weak convergence. The Krylov-Bogolioubov criteria thus implies that there is a measure $\pi_{0}$ such that $\pi_{0} p_{1}=\pi_{0}$, cf. e.g. [26, Theorem 1.10]. It is straightforward to check that $\pi:=\int_{0}^{1} \pi_{0} p_{s} d s$ is invariant w.r.t. $\left(p_{t}\right)$, cf. e.g. [31, Section 3]. Moreover, Assumption 4 implies that any invariant probability measure $\pi^{\star}$ satisfies $\pi^{\star} \in \mathcal{P}_{V}$, cf. e.g. [25, Proposition 4.24], and thus (74) implies that $\pi$ is the only invariant measure. 


\section{Applications}

We demonstrate the applicability of the results from Section 2 .

\subsection{Absolutely continuous measures w.r.t. a normal distribution.}

3.1.1. General setup. Suppose that $\mathcal{G}$ is the covariance operator of a non-degenerate and centered normal distribution $\mathcal{N}(0, \mathcal{G})$ on a separable Hilbert space $(\mathbb{H},\langle\cdot, \cdot\rangle,|\cdot|)$, i.e. $\mathcal{G}$ is trace-class, symmetric and positive-definite. Define a probability measure $\pi$ by (2), where $U: \mathbb{H} \rightarrow \mathbb{R}$ is a given potential which is bounded from below, Fréchet differentiable and for which $x \mapsto \nabla U(x)$ is Lipschitz. We define $b$ in equation (11) as $b(x)=-\mathcal{G} \nabla U(x)$. The results from [24] imply that $\pi$ is an invariant measure for $\left(p_{t}\right)$, i.e. $\pi p_{t}=\pi$ for any $t \geq 0$. We now give sufficient conditions under which the results from Section 2 are applicable in this context.

Remark 1. The article 24] by Hairer, Stuart and Voss considers two different SPDEs for which $\pi$ is a stationary distribution and which can both be used for sampling purposes. The first one is given by

$$
d \tilde{X}_{t}=\Delta \tilde{X}_{t} d t-\nabla U\left(\tilde{X}_{t}\right) d t+\sqrt{2} d \tilde{W}_{t}
$$

where $\left(\tilde{W}_{t}\right)$ is a cylindrical Wiener process over $\mathbb{H}$. The second one is given by (1) and this is the one we study in this article. Formally, the latter equation is obtained from (77) by "preconditioning". The solutions for the equations behave quite differently: While (77) only admits mild solutions in general, strong solutions are possible for (11). Moreover, as pointed out in [24] under reasonable assumptions, the process $\left(\tilde{X}_{t}\right)$ is strong Feller and it is possible to apply classical Harris' theorems to study ergodic properties. In contrast to this, the process $\left(X_{t}\right)$ solving (1) is not strong Feller in general and the study of ergodic properties is more involved.

Fix an orthonormal basis $\left(\mathbf{e}_{k}\right)_{k \in \mathbb{N}_{+}}$of $\mathbb{H}$ such that $\mathcal{G} \mathbf{e}_{k}=\lambda_{k} \mathbf{e}_{k}$ holds for a sequence $\left(\lambda_{k}\right)$ of positive reals satisfying $\sum_{k=1}^{\infty} \lambda_{k}<\infty$. For the sake of simplicity, we assume $\lambda_{k} \downarrow 0$ and $\lambda_{1}=1$. Observe that Theorem 1 and 2 still hold true, if we replace Assumptions 1 and 3 by the slightly more general Assumptions 5 and 6] respectively.

Assumption 5. There are constants $0 \leq H_{h}<1$ and $L_{l}, L_{h}, H_{l} \geq 0$ such that

$$
\left\langle\frac{x^{h}-y^{h}}{\left|x^{h}-y^{h}\right|}, b(x)-b(y)\right\rangle \leq H_{l}\left|x^{l}-y^{l}\right|+H_{h}\left|x^{h}-y^{h}\right|
$$

for any $x, y \in \mathbb{H}$ with $x^{h} \neq y^{h}$ and in the case $x^{l} \neq y^{l}$, we have

$$
\left\langle\frac{x^{l}-y^{l}}{\left|x^{l}-y^{l}\right|}, b(x)-b(y)\right\rangle \leq L_{l}\left|x^{l}-y^{l}\right|+L_{h}\left|x^{h}-y^{h}\right| .
$$

Assumption 6. There are $R \in(0, \infty)$ and $0 \leq M<1$ such that

$$
I_{x^{l} \neq y^{l}}\left\langle\frac{x^{l}-y^{l}}{\left|x^{l}-y^{l}\right|}, b(x)-b(y)\right\rangle+I_{x^{h} \neq y^{h}} \alpha\left\langle\frac{x^{h}-y^{h}}{\left|x^{h}-y^{h}\right|}, b(x)-b(y)\right\rangle \leq M|x-y|_{\alpha}
$$

for any $x, y \in \mathbb{H}$ with $|x-y|_{\alpha} \geq R$. 
In the following we focus on potentials $U: \mathbb{H} \rightarrow \mathbb{R}$ of the form

$$
U(x)=\frac{a}{2}|x|^{2}+m(x)
$$

where $a \geq 0$ and $m: \mathbb{H} \rightarrow \mathbb{R}$ satisfies:

Assumption 7. The function $m$ is bounded from below and Fréchet differentiable. There is $L \geq 1$ such that

$$
|\nabla m(x)-\nabla m(y)| \leq L|x-y| \quad \text { holds true for any } x, y \in \mathbb{H} \text {. }
$$

Lemma 10. Let Assumption $\square$ be true and define $n=\min \left\{k \in \mathbb{N}_{+}: \lambda_{k+1}<\frac{1}{2 L}\right\}$. We consider the splitting $\mathbb{H}=\mathbb{H}^{l} \oplus \mathbb{H}^{h}$ with $\mathbb{H}^{l}=\left\langle\boldsymbol{e}_{1}, \ldots, \boldsymbol{e}_{n}\right\rangle$. In this setting, Assumption 5 is satisfied with

$$
H_{l}=H_{h}=1 / 2, \quad L_{l}=L_{h}=L, \quad \alpha=2(1+L) \quad \text { and } \beta=2 L .
$$

Proof. Let $x, y \in \mathbb{H}$ with $x^{h}-y^{h} \neq 0$. We have

$$
\begin{aligned}
-\left\langle x^{h}-y^{h}, \mathcal{G}(\nabla U(x)-\nabla U(y))\right\rangle= & -a\left\langle x^{h}-y^{h}, \mathcal{G}(x-y)\right\rangle \\
& -\left\langle x^{h}-y^{h}, \mathcal{G}(\nabla m(x)-\nabla m(y))\right\rangle .
\end{aligned}
$$

Observe that $-a\left\langle x^{h}-y^{h}, \mathcal{G}(x-y)\right\rangle \leq 0$. Using Cauchy-Schwarz, we get

$$
\begin{aligned}
\left|\left\langle x^{h}-y^{h}, \mathcal{G}(\nabla m(x)-\nabla m(y))\right\rangle\right| & \leq \lambda_{n+1} L\left|x^{h}-y^{h}\right||x-y| \\
& \leq 1 / 2\left|x^{h}-y^{h}\right||x-y| .
\end{aligned}
$$

This implies (78) with $H_{l}=H_{h}=1 / 2$. Inequality (79) can be argued similarly.

Lemma 11. Assume that there is $\mathcal{R}>0$ such that $\nabla m(x)=0$ for any $|x| \geq \mathcal{R}$. Then Assumption 6 can be satisfied with $M=3 / 4$ and $R=8 L \mathcal{R}$.

Proof. Let $x, y \in \mathbb{H}$ with $|x-y|_{\alpha} \geq R$. The statement is clear if $\min \{|x|,|y|\} \geq \mathcal{R}$. Assume w.l.o.g. that $|x|<\mathcal{R},|y| \geq \mathcal{R}$ and let $z \in \mathbb{H}$ with $|z|=\mathcal{R}$, then

$$
\begin{aligned}
& I_{x^{l} \neq y^{l}}\left\langle\frac{x^{l}-y^{l}}{\left|x^{l}-y^{l}\right|}, b(x)-b(y)\right\rangle+I_{x^{h} \neq y^{h}} \alpha\left\langle\frac{x^{h}-y^{h}}{\left|x^{h}-y^{h}\right|}, b(x)-b(y)\right\rangle \\
\leq & \left|(\mathcal{G}(\nabla m(x)-\nabla m(z)))^{l}\right|+\alpha\left|(\mathcal{G}(\nabla m(x)-\nabla m(z)))^{h}\right| \\
\leq & L|x-z|+\alpha \lambda_{n+1} L|x-z| \leq 2 L \mathcal{R}+2(1+L) \mathcal{R} \leq 3 / 4|x-y|_{\alpha} .
\end{aligned}
$$

Corollary 7. If the assumptions of Lemma 10 and 11 are satisfied, then Theorem 1 holds with $4 c \geq \exp \left(-32 L^{4} \mathcal{R}^{2}\right) /(1+L)$.

We give a sufficient condition for the existence of a Lyapunov function.

Lemma 12. Let Assumption 可 be true and set $V(x)=1+|x|^{2}$. If there are constants $b \in(0, \infty)$ and $0 \leq c<1$ such that

$$
|\nabla m(x)| \leq b+c|x| \quad \text { holds for any } x \in \mathbb{H} \text {, }
$$

then for any $0<\eta<1-c$ there is $C \in(0, \infty)$ such that Assumption 4 is satisfied with $(V, C, \eta)$. 
Proof. We have to find $C$ such that (16) holds true for all $x \in \mathbb{H}$. Observe that,

$$
\begin{aligned}
\mathcal{L} V(x) & =2\langle x,-x-a \mathcal{G} x-\mathcal{G} \nabla m(x)\rangle+\operatorname{trace}(\mathcal{G}) \\
& \leq 2\left(-|x|^{2}+b|x|+c|x|^{2}\right)+\operatorname{trace}(\mathcal{G}) .
\end{aligned}
$$

The claim follows since $c<1$.

We see that Theorem 2 is applicable if the assumptions of Lemma12 are satisfied.

3.1.2. Transition path sampling. We present a concrete sampling context for which the results from the last subsection are applicable. We follow here [24] and consider the $\mathbb{R}^{d}$-valued SDE

$$
d X_{t}=-\nabla_{\mathbb{R}^{d}} W\left(X_{t}\right) d t+d B_{t}, \quad X_{0}=0,
$$

where $\left(B_{t}\right)$ is a $d$-dimensional Brownian motion.

Assumption 8. The potential $W: \mathbb{R}^{d} \rightarrow \mathbb{R}$ is given by

$$
W(x)=\frac{a}{2}|x|^{2}+H(x),
$$

with $a>0$ and $H: \mathbb{R}^{d} \rightarrow \mathbb{R}$ is a $C^{4}$ function for which all $k$-fold partial derivatives, $k \in\{1,2,3,4\}$, satisfy

$$
\left|\partial^{k} H(x)\right||x|^{k-2} \rightarrow 0 \text { for }|x| \rightarrow \infty .
$$

Suppose that we are interested in the law $\pi$ of $\left(X_{t}\right)_{t \in[0,1]}$ conditioned on the event $X_{1}=0$. We describe a typical setup for the above situation. Set $\mathbb{H}=$ $L^{2}\left([0,1], \mathbb{R}^{d}\right)$ and let $\left(\Delta_{0}, D_{0}\right)$ be the self-adjoint Laplacian with Dirichlet boundary condition, i.e. the domain $D_{0}$ is given by all differentiable functions $f$, such that $f^{\prime}$ is absolutely continuous with $f^{\prime \prime} \in L^{2}$ and such that $f(0)=f(1)=0$. Let $\mathcal{G}=-\Delta_{0}^{-1}$. Observe that $\mathbf{e}_{k}=\sqrt{2} \sin (\pi k t), k \in \mathbb{N}$, is an orthonormal basis of $\mathbb{H}$ satisfying $\mathcal{G} \mathbf{e}_{k}=\lambda_{k} \mathbf{e}_{k}$ with $\lambda_{k}=(\pi k)^{-2}$. In particular, the operator $\mathcal{G}$ is trace-class, symmetric and positive definite on $\mathbb{H}$. It is well-known, that the distribution of a standard Brownian Bridge on $\mathbb{H}$ is a centered normal distribution with covariance operator $\mathcal{G}$. Under Assumption 8 one can argue, using Girsanov's theorem and Itô's formula, that the law $\pi$ of $\left(X_{t}\right)_{t \in[0,1]}$ conditioned on $X_{1}=0$ is given by (2), with $U: \mathbb{H} \rightarrow \mathbb{R}$ defined by

$$
U(x)=\frac{1}{2} \int_{0}^{1} \Phi\left(x_{s}\right) d s \text { and } \Phi(x)=\left|\nabla_{\mathbb{R}^{d}} W(x)\right|^{2}+\Delta_{\mathbb{R}^{d}} W(x) .
$$

We refer the reader to [24] for a detailed exposition. It is now a straightforward calculation to check that Lemma 12 is applicable, if Assumption 8 is satisfied.

3.2. Finite-dimensional approximations. In this work we focus on explicit contraction rates for the process (1). In the light of sampling applications one might ask, if it is possible to make related statements about finite-dimensional approximations. We shortly argue, that this is indeed the case.

Suppose that Assumptions 1, 2, and 3 are true. Let $\mathbb{H}^{l}$ be of dimension $n \in \mathbb{N}_{+}$. Fix some $d>n$ and write $\mathbb{H}^{d}=\left\langle\mathbf{e}_{1}, \ldots, \mathbf{e}_{d}\right\rangle$ for the subspace spanned by the first $d$ basis vectors. Given $x \in \mathbb{H}$, we write $x^{d}$ for the orthogonal projection onto 
$\mathbb{H}^{d}$. Let $\left(X_{t}\right)$ be a solution of (11) with $X_{0}=x_{0}$. A straightforward $d$-dimensional approximation $\left(\tilde{X}_{t}\right)$ is given by the solution of the $\mathrm{SDE}$

$$
d \tilde{X}_{t}=-\tilde{X}_{t} d t+b^{d}\left(\tilde{X}_{t}\right) d t+\sqrt{2} d W_{t}^{d}, \quad \tilde{X}_{0}=x_{0}^{d}
$$

A similar approximation is e.g. considered in [9]. Observe that the non-linearity $x \mapsto b^{d}(x)$ satisfies Assumptions 1 and 3 on the space $\mathbb{H}^{d}$ with the same constants as $x \mapsto b(x)$ on $\mathbb{H}$. In particular, we can apply Theorem 1 to equation (85) and see that the corresponding Markov kernels satisfy a Kantorovich contraction with a dimension-independent and explicit contraction rate. A related statement holds true for Theorem 2. We remark that the unique invariant measure $\pi^{d}$ for (85) does in general not agree with the invariant measure $\pi$ of (1). A study of the approximation error can be found in [9].

It might also be possible to use the presented results to make statements about the speed of convergence of time-discrete approximations of (85), e.g. Euler approximations. There are at least two different approaches to this question: The first possibility is to implement a similar coupling strategy directly for Markov chains. We refer in this context to the forthcoming work [11. The second possibility is to interpret the approximation as a perturbation of the original equation, see [45, 40, 41, 44, 8, 10] and the references therein. Nevertheless, the last question goes beyond the scope of this work.

\section{ACKNOWLEDGEMENT}

I want to thank my supervisor A. Eberle for suggesting the topic, for his support and helpful advice. I am grateful for several fruitful discussions with A. Guillin on related topics. Moreover, I want to thank M. Hairer for his hospitality during a stay at the University of Warwick and for pointing out the connection of my work to the 2D Navier-Stokes equation.

\section{REFERENCES}

[1] J. Bricmont, A. Kupiainen, and R. Lefevere. Ergodicity of the 2D Navier-Stokes equations with random forcing. Comm. Math. Phys., 224(1):65-81, 2001. Dedicated to Joel L. Lebowitz.

[2] J. Bricmont, A. Kupiainen, and R. Lefevere. Exponential mixing of the 2D stochastic NavierStokes dynamics. Comm. Math. Phys., 230(1):87-132, 2002.

[3] M. F. Chen and F.-Y. Wang. Estimation of the first eigenvalue of second order elliptic operators. J. Funct. Anal., 131(2):345-363, 1995.

[4] M.-F. Chen and F.-Y. Wang. Estimation of spectral gap for elliptic operators. Trans. Amer. Math. Soc., 349(3):1239-1267, 1997.

[5] G. Da Prato, A. Debussche, and L. Tubaro. Coupling for some partial differential equations driven by white noise. Stochastic Process. Appl., 115(8):1384-1407, 2005.

[6] G. Da Prato and J. Zabczyk. Ergodicity for infinite-dimensional systems, volume 229 of London Mathematical Society Lecture Note Series. Cambridge University Press, Cambridge, 1996.

[7] G. Da Prato and J. Zabczyk. Stochastic equations in infinite dimensions, volume 152 of Encyclopedia of Mathematics and its Applications. Cambridge University Press, Cambridge, second edition, 2014. 
[8] A. S. Dalalyan. Theoretical guarantees for approximate sampling from smooth and logconcave densities. ArXiv e-prints, December 2014.

[9] M. Dashti and A. M. Stuart. The Bayesian Approach To Inverse Problems. ArXiv e-prints, February 2013.

[10] A. Durmus and E. Moulines. Non-asymptotic convergence analysis for the Unadjusted Langevin Algorithm. ArXiv e-prints, July 2015.

[11] A. Eberle. Quantitative contraction rates for Markov chains on continuous state spaces. preprint.

[12] A. Eberle. Reflection couplings and contraction rates for diffusions. Probability Theory and Related Fields, pages 1-36, 2015.

[13] A. Eberle, A. Guillin, and R. Zimmer. Quantitative Harris type theorems for diffusions and McKean-Vlasov processes. ArXiv e-prints, June 2016.

[14] Andreas Eberle. Reflection coupling and Wasserstein contractivity without convexity. C. R. Math. Acad. Sci. Paris, 349(19-20):1101-1104, 2011.

[15] L. Gawarecki and V. Mandrekar. Stochastic differential equations in infinite dimensions with applications to stochastic partial differential equations. Probability and its Applications (New York). Springer, Heidelberg, 2011.

[16] M. Hairer. Exponential mixing properties of stochastic PDEs through asymptotic coupling. Probab. Theory Related Fields, 124(3):345-380, 2002.

[17] M. Hairer and J. C. Mattingly. Ergodic properties of highly degenerate 2D stochastic NavierStokes equations. C. R. Math. Acad. Sci. Paris, 339(12):879-882, 2004.

[18] M. Hairer and J. C. Mattingly. Ergodicity of the 2D Navier-Stokes equations with degenerate stochastic forcing. Ann. of Math. (2), 164(3):993-1032, 2006.

[19] M. Hairer and J. C. Mattingly. Spectral gaps in Wasserstein distances and the 2D stochastic Navier-Stokes equations. Ann. Probab., 36(6):2050-2091, 2008.

[20] M. Hairer and J. C. Mattingly. A theory of hypoellipticity and unique ergodicity for semilinear stochastic PDEs. Electron. J. Probab., 16:no. 23, 658-738, 2011.

[21] M. Hairer and J. C. Mattingly. Yet another look at Harris' ergodic theorem for Markov chains. In Seminar on Stochastic Analysis, Random Fields and Applications VI, volume 63 of Progr. Probab., pages 109-117. Birkhäuser/Springer Basel AG, Basel, 2011.

[22] M. Hairer, J. C. Mattingly, and M. Scheutzow. Asymptotic coupling and a general form of Harris' theorem with applications to stochastic delay equations. Probab. Theory Related Fields, 149(1-2):223-259, 2011.

[23] M. Hairer, A: M. Stuart, and S. J. Vollmer. Spectral gaps for a Metropolis-Hastings algorithm in infinite dimensions. Ann. Appl. Probab., 24(6):2455-2490, 2014.

[24] M. Hairer, A. M. Stuart, and J. Voss. Analysis of SPDEs arising in path sampling. II. The nonlinear case. Ann. Appl. Probab., 17(5-6):1657-1706, 2007.

[25] Martin Hairer. Ergodic properties of markov processes. Lecture notes, Univ. Warwick. http://www.hairer.org/notes/Markov.pdf, 2006.

[26] Martin Hairer. Convergence of Markov processes. Lecture notes, Univ. Warwick. http://www.hairer.org/notes/Convergence.pdf, 2016.

[27] T. E. Harris. The existence of stationary measures for certain Markov processes. In Proceedings of the Third Berkeley Symposium on Mathematical Statistics and Probability, 19541955, vol. II, pages 113-124. University of California Press, Berkeley and Los Angeles, 1956.

[28] Aldéric Joulin and Yann Ollivier. Curvature, concentration and error estimates for Markov chain Monte Carlo. Ann. Probab., 38(6):2418-2442, 2010.

[29] O. Kallenberg. Foundations of modern probability. Probability and its Applications (New York). Springer-Verlag, New York, second edition, 2002.

[30] Rafail Khasminskii. Stochastic stability of differential equations, volume 66 of Stochastic Modelling and Applied Probability. Springer, Heidelberg, second edition, 2012. With contributions by G. N. Milstein and M. B. Nevelson. 
[31] Tomasz Komorowski and Anna Walczuk. Central limit theorem for markov processes with spectral gap in the wasserstein metric. Stochastic Processes and their Applications, 122(5):2155-2184, 2012.

[32] S. Kuksin and A. Shirikyan. Stochastic dissipative PDEs and Gibbs measures. Comm. Math. Phys., 213(2):291-330, 2000.

[33] S. Kuksin and A. Shirikyan. A coupling approach to randomly forced nonlinear PDE's. I. Comm. Math. Phys., 221(2):351-366, 2001.

[34] G. Leha and G. Ritter. On diffusion processes and their semigroups in Hilbert spaces with an application to interacting stochastic systems. Ann. Probab., 12(4):1077-1112, 1984.

[35] T. Lindvall and L. C. G. Rogers. Coupling of multidimensional diffusions by reflection. Ann. Probab., 14(3):860-872, 1986.

[36] N. Masmoudi and L.-S. Young. Ergodic theory of infinite dimensional systems with applications to dissipative parabolic PDEs. Comm. Math. Phys., 227(3):461-481, 2002.

[37] J. C. Mattingly. Exponential convergence for the stochastically forced Navier-Stokes equations and other partially dissipative dynamics. Comm. Math. Phys., 230(3):421-462, 2002.

[38] J. C. Mattingly, N. S. Pillai, and A. M. Stuart. Diffusion limits of the random walk Metropolis algorithm in high dimensions. Ann. Appl. Probab., 22(3):881-930, 2012.

[39] S. P. Meyn and R. L. Tweedie. Markov chains and stochastic stability. Communications and Control Engineering Series. Springer-Verlag London, Ltd., London, 1993.

[40] A. Yu. Mitrophanov. Stability and exponential convergence of continuous-time Markov chains. J. Appl. Probab., 40(4):970-979, 2003.

[41] N. S. Pillai and A. Smith. Ergodicity of Approximate MCMC Chains with Applications to Large Data Sets. ArXiv e-prints, May 2014.

[42] M. Reed and B. Simon. Methods of modern mathematical physics. I. Academic Press, Inc. [Harcourt Brace Jovanovich, Publishers], New York, second edition, 1980. Functional analysis.

[43] G. O. Roberts and J. S. Rosenthal. Quantitative bounds for convergence rates of continuous time Markov processes. Electron. J. Probab., 1:no. 9, approx. 21 pp. (electronic), 1996.

[44] D. Rudolf and N. Schweizer. Perturbation theory for Markov chains via Wasserstein distance. ArXiv e-prints, March 2015.

[45] T. Shardlow and A. M. Stuart. A perturbation theory for ergodic Markov chains and application to numerical approximations. SIAM J. Numer. Anal., 37(4):1120-1137, 2000.

[46] A. M. Stuart, Jochen Voss, and Petter Wiberg. Fast communication conditional path sampling of SDEs and the Langevin MCMC method. Commun. Math. Sci., 2(4):685-697, 2004.

[47] A. Yu. Veretennikov and A. M. Kulik. The extended Poisson equation for weakly ergodic Markov processes. Teor. Imovīr. Mat. Stat., (85):22-38, 2011.

[48] C. Villani. Optimal transport, volume 338 of Grundlehren der Mathematischen Wissenschaften [Fundamental Principles of Mathematical Sciences]. Springer-Verlag, Berlin, 2009. Old and new.

[49] F.-Y. Wang. Asymptotic couplings by reflection and applications for nonlinear monotone SPDES. Nonlinear Anal., 117:169-188, 2015.

[50] E. Weinan and D. Liu. Gibbsian dynamics and invariant measures for stochastic dissipative PDEs. J. Statist. Phys., 108(5-6):1125-1156, 2002. Dedicated to David Ruelle and Yasha Sinai on the occasion of their 65 th birthdays.

[51] E. Weinan, J. C. Mattingly, and Ya. Sinai. Gibbsian dynamics and ergodicity for the stochastically forced Navier-Stokes equation. Comm. Math. Phys., 224(1):83-106, 2001. Dedicated to Joel L. Lebowitz.

Institute for Applied Mathematics, University of Bonn, Endenicher Allee 60, 53115 Bonn, Germany

E-mail address: Raphael.Zimmer@uni-bonn.de 